\title{
Maximum-principle-satisfying space-time conservation element and solution element scheme applied to compressible multifluids
}

\author{
Hua Shen ${ }^{1}$, Chih-Yung Wen ${ }^{2}$, Matteo Parsani ${ }^{1}$, Chi-Wang Shu ${ }^{3}$
}

\begin{abstract}
A maximum-principle-satisfying space-time conservation element and solution element (CE/SE) scheme is constructed to solve a reduced five-equation model coupled with the stiffened equation of state for compressible multifluids. We first derive a sufficient condition for CE/SE schemes to satisfy maximum-principle when solving a general conservation law. And then we introduce a slope limiter to ensure the sufficient condition which is applicative for both central and upwind $\mathrm{CE} / \mathrm{SE}$ schemes. Finally, we implement the upwind maximum-principle-satisfying $\mathrm{CE} / \mathrm{SE}$ scheme to solve the volume-fraction-based five-equation model for compressible multifluids. Several numerical examples are carried out to carefully examine the accuracy, efficiency, conservativeness and maximum-principle-satisfying property of the proposed approach.
\end{abstract}

Keywords: maximum-principle-satisfying scheme; space-time conservation element and solution element (CE/SE) method; upwind scheme; compressible multifluids; five-equation model.

\section{Introduction}

An important property of the entropy solution of scalar conservation laws is satisfying a strict maximum (or bound-preserving) principle [1], that is, if the initial value of the conservative quantity is in the range of $[m, M]$, the solution will remain in this range at any time and position. Numerical schemes with this property are very 
useful for some applications (e.g., flows consisting of several fluid components). When using Eulerian diffusive interface models to treat such flows, the interface can be represented by mass fractions $[2,3]$, the ratio of specific heats $\gamma$ (or its function) [3-5] or volume fractions [6-8]. In addition, the interface can be evolved by a scalar advection equation coupled to the compressible Euler or Navier-Stokes equations. The interface between different fluids is virtually a contact discontinuity. To simulate this problem accurately, not only sharp interface capturing is required, but also the maximum-principle-satisfying property is desired, especially for fluids with large difference in their densities or in their thermodynamic properties. If the volume or mass fraction of any component becomes negative, the numerical solution may blowup. If we directly force the value to be positive (e.g., by "clipping" the component that becomes negative), the scheme will become non-conservative.

Canonical total variation diminishing (TVD) schemes [9] strictly satisfy maximum principle properties but their accuracy will reduce to first-order at smooth extrema [10] which may cause excessive dissipation near the interface. Zhang et al. [1] constructed uniformly high-order accurate schemes satisfying a strict maximum principle by employing a general limiter for finite volume schemes (e.g., essentially non-oscillatory (ENO) or weighted ENO (WENO) schemes) or discontinuous Galerkin (DG) methods. The space-time conservation element and solution element (CE/SE) method, originally developed by Chang and co-workers [11-13], is a unique central second-order scheme which strictly follows the space-time conservation law. Later on, Bilyeu et al. [14] and Shen et al. [15] developed high-order CE/SE schemes on unstructured triangular meshes and hybrid meshes, respectively. These CE/SE schemes capture shock efficiently and accurately, but their resolution for individual contact discontinuities (material interfaces) is unsatisfactory [16-17]. Qamar et al. have performed simulations of compressible two-fluid problems using the central $\mathrm{CE} / \mathrm{SE}$ scheme by [18]. Therein, it is found that interfaces are diffusive. To overcome this disadvantage, Shen et al. $[16,17]$ recently proposed a characteristic-based (or an upwind) $\mathrm{CE} / \mathrm{SE}$ scheme that preserves the space-time conservativeness of the original $\mathrm{CE} / \mathrm{SE}$ scheme, while significantly improving its resolution for material interfaces; 
however, their schemes did not satisfy the maximum principle. In fact, one can observe from Fig. 3 of Ref. [16] that the upwind CE/SE scheme is more stable than are the central ones, but its solution is not strictly in the range of the initial conditions. Guaranteeing the maximum-principle-satisfying property of the CE/SE method (even for second-order schemes) is not straightforward, because its construction process, especially the treatment of time, is unique. In conventional finite volume or finite difference schemes, space and time are treated separately using the method of lines approach. Cell averages or point values are first updated, and then spatial reconstruction techniques are used to calculate derivatives in terms of cell averages or point values. In second-order TVD schemes, a limiter with TVD property using for the slope (first-order derivative) reconstruction can make the schemes satisfy the maximum principle [1]. Such a property is then maintained using strong stability preserving (SSP) time discretizations [19]. However, in CE/SE space and time are unified and treated in the same manner. The mesh quantities and their derivatives are treated as independent variables and are updated simultaneously by individual time marching schemes. To date, no maximum-principle-satisfying CE/SE scheme has been proposed.

In this paper, we present a maximum-principle-satisfying $\mathrm{CE} / \mathrm{SE}$ scheme for scalar conservation laws by adopting the limiter proposed by Zhang and Shu [1]. Although in theory this idea could be applied to both central and upwind CE/SE schemes, we limit our focus to the upwind CE/SE scheme and its application to solve a reduced five-equation model [4-8] for compressible multifluids.

We begin by reviewing how CE/SE schemes are typically formulated, and then in Section 3, we derive sufficient conditions for one-dimensional (1D) and two-dimensional (2D) maximum-principle-satisfying CE/SE schemes. The maximum-principle-satisfying upwind CE/SE scheme coupled with the HLLC-CE/SE Euler solver is extended to compressible multifluids in Section 4, and in Section 5, several numerical examples are performed to verify the accuracy and completeness of the maximum-principle-satisfying property of the newly developed scheme. Concluding remarks are given in Section 6. 


\section{A brief introduction to the CE/SE method}

\subsection{The basic idea behind space-time conservation}

Consider the differential form of the scalar conservation laws

$$
\frac{\partial u}{\partial t}+\nabla \cdot \mathbf{F}(u)=0
$$

where $u$ and $\mathbf{F}$ are the conservative quantity and corresponding spatial flux vector, respectively. Using the Gauss divergence theorem, the differential equation can be written in the integral form as

$$
\int_{S(V)} \mathbf{h} \cdot \mathbf{d s}=0
$$

where $V$ can be an arbitrary closed space-time region, $S(V)$ the boundary of $V, \mathbf{h}=(\mathbf{F}, u)$ the space-time flux vector and $\mathbf{d s} \equiv \mathrm{d} \sigma \mathbf{n}$, in which $\mathrm{d} \sigma$ and $\mathbf{n}$ are the size and unit outward normal vector of the corresponding boundary element on $S(V)$, respectively. Eq. (2) is an integral form of Eq. (1), implying that the general space-time conservation law does not depend on the dimension of the problem. The CE/SE method strictly follows this space-time conservation law to construct numerical schemes. Although the basic idea of different CE/SE schemes is identical, the specific forms are closely related to the definition of the so-called CE and SE. CE is a space-time control volume on which the conservation law Eq. (2) is implemented. The configuration of $\mathrm{CE}$ determines the discrete form of the space-time conservation law. Meanwhile, SE is used to solve the fluxes involved in the discrete conservation law. Usually, the fluxes in each SE are approximated by Taylor expansion [11].

\subsection{One dimensional CE/SE schemes}

For the 1D case, we consider uniform mesh grids. Definitions of CE and SE for the central CE/SE scheme [11] are depicted in Fig. 1 (a). Implementing the conservation law Eq. (2) on $\mathrm{CE}_{j}^{n,-}$ and $\mathrm{CE}_{j}^{n,+}$, leads to the following equations,

$$
\begin{aligned}
& U_{L}^{*} \frac{\Delta x}{2}=U_{L} \frac{\Delta x}{2}+\left(F_{L}-F_{C}\right) \frac{\Delta t}{2}, \\
& U_{R}^{*} \frac{\Delta x}{2}=U_{R} \frac{\Delta x}{2}+\left(F_{C}-F_{R}\right) \frac{\Delta t}{2},
\end{aligned}
$$

where, $U_{L}{ }^{*}, U_{R}{ }^{*}, U_{L}, U_{R}, F_{L}, F_{R}$ and $F_{C}$ are the average fluxes ( $U$ is considered as the temporal flux) through $\mathrm{DF}, \mathrm{FC}, \mathrm{AE}, \mathrm{EB}, \mathrm{AD}, \mathrm{BC}$ and $\mathrm{EF}$, respectively. With the aid of 
the first-order Taylor expansion in the associated SEs, the average fluxes can be calculated as

$$
\begin{aligned}
U_{L}^{*} & =u_{j}^{n}-\left(u_{x}\right)_{j}^{n} \frac{\Delta x}{4}, U_{R}^{*}=u_{j}^{n}+\left(u_{x}\right)_{j}^{n} \frac{\Delta x}{4}, F_{C}=f_{j}^{n}-\frac{\Delta t}{4}\left(f_{t}\right)_{j}^{n}, \\
U_{L} & =u_{j-1 / 2}^{n-1 / 2}+\left(u_{x}\right)_{j-1 / 2}^{n-1 / 2} \frac{\Delta x}{4}, F_{L}=f_{j-1 / 2}^{n-1 / 2}+\frac{\Delta t}{4}\left(f_{t}\right)_{j-1 / 2}^{n-1 / 2}, \\
U_{R} & =u_{j+1 / 2}^{n-1 / 2}-\left(u_{x}\right)_{j+1 / 2}^{n-1 / 2} \frac{\Delta x}{4}, F_{R}=f_{j+1 / 2}^{n-1 / 2}+\frac{\Delta t}{4}\left(f_{t}\right)_{j+1 / 2}^{n-1 / 2} .
\end{aligned}
$$

Substituting these fluxes into Eqs. (3) and (4) yields, the following time marching schemes for $u_{j}^{n}$ and $\left(u_{x}\right)_{j}{ }^{n}$

$$
u_{j}^{n}=\frac{1}{2}\left(U_{L}+U_{R}\right)+\frac{\Delta t}{2 \Delta x}\left(F_{L}-F_{R}\right),
$$

and

$$
\left(u_{x}\right)_{j}^{n} \frac{\Delta x}{4}=\frac{1}{2}\left(U_{R}-U_{L}\right)+\frac{\Delta t}{2 \Delta x}\left(2 F_{C}-F_{L}-F_{R}\right) .
$$

The temporal derivative is calculated using the Cauchy-Kovalewski procedure, which is based on repeated differentiation of the governing partial differential equation and has been employed in the original ENO [20] and ADER schemes [21]. This is the so called ' $a$ ' scheme [11], which is a non-dissipative scheme. To capture shocks, appropriate dissipation has to be introduced to tame numerical oscillations. In the ' $a-\alpha$ ' CE/SE scheme [11], the time marching scheme of $\left(u_{x}\right)_{j}^{n}$ is replaced by a limited central difference procedure. The dissipation can be adjusted by the parameter ' $\alpha$ ' that appears in the weighted average function; however, when the Courant-Friedrichs-Lewy (CFL) number is small (e.g., smaller than 0.1), the numerical dissipation of the ' $a-\alpha$ ' scheme is excessive. To resolve this limitation, Chang [22] proposed a Courant-number-insensitive (CNI) CE/SE scheme which used the local CFL number to adjust the dissipation. When the CFL number equals to 1 , the CNI scheme is equivalent to the dissipative ' $a-\alpha$ ' scheme, and when the CFL number decreases, the CNI scheme gradually approaches the non-dissipative ' $a$ ' scheme to reduce dissipation. As a consequence, the numerical dissipation can be efficiently controlled.

Most recently, Shen et al. [16] proposed a characteristic CE/SE scheme of which $\mathrm{CE}$ and SE are illustrated by Fig. 1(b). In the characteristic CE/SE scheme, the definition of the $\mathrm{CE}$ is identical to that of the central CE/SE scheme. Hence, the same 
time marching approach for $u_{j}^{n}$ and $\left(u_{x}\right)_{j}^{n}$ (i.e. Eqs. (6) and (7)) was established by implementing the space-time conservation law on the CE. In the new definition, (DF \& FC), (AE \& AD) and (EB \& BC) are also affiliated to $\mathrm{SE}_{j}^{n}, \mathrm{SE}_{j-1 / 2}^{n-1 / 2}$ and $\mathrm{SE}_{j+1 / 2}^{n-1 / 2}$, respectively. Hence the fluxes through them are continuous and also calculated by the Taylor expansion in the associated SE (i.e., Eq. (5)), but EF is located between $\mathrm{SE}_{j-1 / 2}^{n-1 / 2}$ and $\mathrm{SE}_{j+1 / 2}^{n-1 / 2}$. As a result, the physical quantities across $\mathrm{EF}$ may be discontinuous due to the piece wise linear assumption. Instead of directly using Taylor expansion in $\mathrm{SE}_{j}^{n}$, we use an upwind procedure to calculate the flux through $\mathrm{EF}$ (i.e., $F_{C}$ ). Note that $F_{C}$ only explicitly appears in the time marching scheme of $\left(u_{x}\right)_{j}{ }^{n}$. Thus the only difference between non-dissipative ' $a$ ' and upwind CE/SE schemes is the time marching scheme for derivatives in terms of $F_{C}$. Since some dissipation is introduced by the upwind procedure, the upwind CE/SE scheme is a practical scheme with no other modifications. To suppress spurious oscillations in problems characterized by very strong discontinuities, the WBAP limiter [23] has been used. The upwind CE/SE scheme inherits the original form of the ' $a$ ' scheme and preserves the beauty of the space-time conservativeness. In addition, it is essentially a CNI scheme and can accurately capture shocks and material interfaces. More detailed descriptions about the 1D upwind CE/SE scheme can be found in [16].

\subsection{Two dimensional CE/SE schemes}

The original 2D central CE/SE scheme proposed by Chang et al. [12] relies on triangular meshes. Later, two other approaches were proposed using quadrilateral meshes $[13,24]$. The main difference between the two approaches is the treatment of the mesh. In the approach presented in [13], the solution is only updated at the cell centers as the original scheme does, but in the approach in [24], the solution is updated alternatively between the cell centers and cell vertices. The upwind CE/SE scheme has been recently extended to quadrilateral meshes [17] using this second approach. 
Here, we only summarize the CE/SE schemes for the uniform rectangular meshes, as shown in Fig. 2 (a). For convenience, the point $\left(x_{a}, y_{b}, t_{c}\right)$ is denoted as $P_{a, b}^{c}(a, b$, $c=0,1 / 2,1,3 / 2 \ldots)$. The solution is updated alternatively between cell vertices " $\circ$ " and cell centers " $\Delta$ ". For clarity of presentation, but without loss of generality, the definitions of $\mathrm{SE}_{i-1 / 2, j-1 / 2}^{n-1 / 2}$ and $\mathrm{CE}_{i, j}^{n}$ are shown in Fig. 2 (b) and (c), respectively. Implementing the conservation law expressed by Eq. (2) on $\mathrm{CE}_{L D}, \mathrm{CE}_{R D}, \mathrm{CE}_{R U}$ and $\mathrm{CE}_{L U}$ (the subscripts follow the rule of $L$ : Left; $R$ : Right, $D$ : Lower, $U$ : Upper and C: Center), yields the following four equations

$$
\begin{aligned}
& U_{L D}^{*} \frac{\Delta x \Delta y}{4}=\left(u-u_{x} \frac{\Delta x}{4}-u_{y} \frac{\Delta y}{4}\right)_{i, j}^{n} \frac{\Delta x \Delta y}{4} \\
& =U_{L D} \frac{\Delta x \Delta y}{4}+\left(F_{L D}-F_{C D}\right) \frac{\Delta y \Delta t}{4}+\left(G_{D L}-G_{C L}\right) \frac{\Delta x \Delta t}{4}, \\
& U_{R D}^{*} \frac{\Delta x \Delta y}{4}=\left(u+u_{x} \frac{\Delta x}{4}-u_{y} \frac{\Delta y}{4}\right)_{i, j}^{n} \frac{\Delta x \Delta y}{4} \\
& =U_{R D} \frac{\Delta x \Delta y}{4}+\left(F_{C D}-F_{R D}\right) \frac{\Delta y \Delta t}{4}+\left(G_{D R}-G_{C R}\right) \frac{\Delta x \Delta t}{4}, \\
& U_{R U}^{*} \frac{\Delta x \Delta y}{4}=\left(u+u_{x} \frac{\Delta x}{4}+u_{y} \frac{\Delta y}{4}\right)_{i, j}^{n} \frac{\Delta x \Delta y}{4} \\
& =U_{R U} \frac{\Delta x \Delta y}{4}+\left(F_{C U}-F_{R U}\right) \frac{\Delta y \Delta t}{4}+\left(G_{C R}-G_{U R}\right) \frac{\Delta x \Delta t}{4}, \\
& U_{L U}^{*} \frac{\Delta x \Delta y}{4}=\left(u-u_{x} \frac{\Delta x}{4}+u_{y} \frac{\Delta y}{4}\right)_{i, j}^{n} \frac{\Delta x \Delta y}{4} \\
& =U_{L U} \frac{\Delta x \Delta y}{4}+\left(F_{L U}-F_{C U}\right) \frac{\Delta y \Delta t}{4}+\left(G_{C L}-G_{U L}\right) \frac{\Delta x \Delta t}{4} .
\end{aligned}
$$

In these four equations, $u_{i, j}^{n},\left(u_{x}\right)_{i, j}^{n}$ and $\left(u_{y}\right)_{i, j}^{n}$ are the three unknowns, and the superscript ' $*$ ' represent the corresponding values at the new time level. The average fluxes through the corresponding boundary face of $\mathrm{CE}_{i, j}^{n}$ are demonstrated in Fig. 2 (d). According to the definition of SE, $\left(U_{L D}, F_{L D} \& G_{D L}\right),\left(U_{R D}, F_{R D} \& G_{D R}\right),\left(U_{R U}\right.$, $\left.F_{R U} \& G_{U R}\right)$ and $\left(U_{L U}, F_{L U} \& G_{U L}\right)$ can be calculated by the Taylor expansions from $P_{i-1 / 2, j-1 / 2}^{n-1 / 2}, \quad P_{i+1 / 2, j-1 / 2}^{n-1 / 2}, \quad P_{i+1 / 2, j+1 / 2}^{n-1 / 2}$ and $P_{i-1 / 2, j+1 / 2}^{n-1 / 2}$, respectively. However, the fluxes $\left(F_{C D}\right.$, 
$F_{C U}, G_{C L}$ and $\left.G_{C R}\right)$ across the inner boundaries of $\mathrm{CE}_{i, j}^{n}$ may be discontinuous, and they can be calculated using an upwind procedure. Adding the four equations together, one derives the time marching scheme for $u_{i, j}^{n}$ as

$$
\begin{aligned}
& u_{i, j}^{n}=\frac{1}{4}\left(U_{L D}+U_{R D}+U_{R U}+U_{L U}\right)+\frac{1}{4} \frac{\Delta t}{\Delta x}\left(F_{L D}+F_{L U}-F_{R D}-F_{R U}\right) \\
& +\frac{1}{4} \frac{\Delta t}{\Delta y}\left(G_{D L}+G_{D R}-G_{U L}-G_{U R}\right)
\end{aligned}
$$

which represents the balancing of fluxes through the whole $\mathrm{CE}_{i, j}^{n}$. Similar to the $1 \mathrm{D}$ scheme, no the upwind fluxes appear explicitly in Eq. (12). Eqs. (8) (11) form an over-determined system of equations (i.e., two values for each derivative are available). We use the WBAP limiter to obtain a unique solution. A detailed description of the 2D upwind CE/SE scheme is available in Ref. [17]. For the central $\mathrm{CE} / \mathrm{SE}$ scheme, the time marching scheme of $u$ is the same as Eq. (12). The derivatives are computed by a weighted central difference [13, 24].

\section{Maximum-Principle-Satisfying CE/SE scheme}

\subsection{Sufficient conditions for maximum-principle-satisfying schemes}

Consider an explicit scheme in the general form

$$
u_{j}^{n+1}=H\left(u_{i_{1}}^{n}, u_{i_{2}}^{n} \ldots u_{i_{N}}^{n}\right) .
$$

Assuming $u_{i_{1}}^{n}, u_{i_{2}}^{n} \ldots u_{i_{N}}^{n} \in[m, M]$, two sufficient conditions for $u_{j}^{n+1} \in[m, M]$ can be expressed as [1]

Condition 1:

$$
H(m, m \ldots m)=m, H(M, M \ldots M)=M
$$

Condition 2:

$$
\frac{\partial H}{\partial u_{i_{n}}} \geq 0, n=1 \sim N .
$$

\subsection{One dimensional maximum-principle-satisfying CE/SE scheme}

In the CE/SE method, mesh quantities and their derivatives are updated as independent variables. However, we are only concerned with a range of mesh 
quantities. Substituting the fluxes in Eq. (5) into the updating scheme for mesh quantities (i.e. Eq. (6)), one derives

$$
\begin{aligned}
& u_{j}^{n}=\frac{1}{2}\left[u_{j-1 / 2}^{n-1 / 2}+\frac{\Delta x}{4}\left(u_{x}\right)_{j-1 / 2}^{n-1 / 2}+u_{j+1 / 2}^{n-1 / 2}-\frac{\Delta x}{4}\left(u_{x}\right)_{j+1 / 2}^{n-1 / 2}\right] \\
& +\frac{\lambda}{2}\left[f_{j-1 / 2}^{n-1 / 2}+\frac{\Delta t}{4}\left(f_{t}\right)_{j-1 / 2}^{n-1 / 2}-f_{j+1 / 2}^{n-1 / 2}-\frac{\Delta t}{4}\left(f_{t}\right)_{j+1 / 2}^{n-1 / 2}\right] .
\end{aligned}
$$

In the $\mathrm{CE} / \mathrm{SE}$ method, the chain rule is employed to compute the derivatives of fluxes and the temporal derivative of $u$ is calculated by the Cauchy-Kovalewski procedure. Therefore, Eq. (16) can be further written as

$$
\begin{aligned}
& u_{j}^{n}=\frac{1}{2}\left[u_{j-1 / 2}^{n-1 / 2}+\frac{\Delta x}{4}\left(u_{x}\right)_{j-1 / 2}^{n-1 / 2}+u_{j+1 / 2}^{n-1 / 2}-\frac{\Delta x}{4}\left(u_{x}\right)_{j+1 / 2}^{n-1 / 2}\right] \\
& +\frac{\lambda}{2}\left[f_{j-1 / 2}^{n-1 / 2}-\frac{\Delta t}{4}\left(f_{u}\right)^{2}\left(u_{x}\right)_{j-1 / 2}^{n-1 / 2}-f_{j+1 / 2}^{n-1 / 2}+\frac{\Delta t}{4}\left(f_{u}\right)^{2}\left(u_{x}\right)_{j+1 / 2}^{n-1 / 2}\right],
\end{aligned}
$$

where $\lambda=\Delta t / \Delta x$ and the Jacobian $f_{u}=\partial f / \partial u$. As we see, some derivatives appear in this scheme. This makes it difficult to check its maximum-principle-satisfying property, because it is difficult to estimate the range of the derivatives. We can use a specific case to demonstrate that $\mathrm{CE} / \mathrm{SE}$ schemes available in the literature do not generally satisfy the maximum principle. Assuming $u \in[0,1], f(u)=u$ and $u_{j-1 / 2}^{n-1 / 2}=u_{j+1 / 2}^{n-1 / 2}=\left(u_{x}\right)_{j+1 / 2}^{n-1 / 2}=0$, Eq. (16) gives $u_{j}^{n}=\frac{\Delta x}{8}\left(1-\lambda^{2}\right)\left(u_{x}\right)_{j-1 / 2}^{n-1 / 2}$. If $\left(u_{x}\right)_{j-1 / 2}^{n-1 / 2}$ is negative, then $u_{j}^{n}$ will always be negative under the CFL condition $\lambda \leqslant 1$. Therefore, additional conditions are required for maximum-principle-satisfying CE/SE scheme.

To directly use the two conditions in Sec. 3.1, we rewrite the derivative terms as

$$
\left(u_{x}\right)_{j \pm 1 / 2} \frac{\Delta x}{2}=u_{j \pm 1 / 2}^{C}-u_{j \pm 1 / 2}^{L}=u_{j \pm 1 / 2}^{R}-u_{j \pm 1 / 2}^{C},
$$

where the superscripts $L, C$ and $R$ represent values at the left, center and right, respectively, of the corresponding cell. To simplify the notation, we drop the superscript $n$-1/2. Inserting Eq. (18) into Eq. (17) yields

$$
\begin{aligned}
& u_{j}^{n}=\frac{1}{4}\left[u_{j-1 / 2}^{C}+u_{j-1 / 2}^{R}+u_{j+1 / 2}^{L}+u_{j+1 / 2}^{C}\right]+\frac{\lambda}{2} f_{j-1 / 2}^{C}-\frac{\lambda^{2}}{4}\left(f_{u}^{C}\right)_{j-1 / 2}^{2}\left(u_{j-1 / 2}^{R}-u_{j-1 / 2}^{C}\right) \\
& -\frac{\lambda}{2} f_{j+1 / 2}^{C}+\frac{\lambda^{2}}{4}\left(f_{u}^{C}\right)_{j+1 / 2}^{2}\left(u_{j+1 / 2}^{C}-u_{j+1 / 2}^{L}\right)=H\left[u_{j-1 / 2}^{C}, u_{j-1 / 2}^{R}, u_{j+1 / 2}^{L}, u_{j+1 / 2}^{C}\right] .
\end{aligned}
$$


It is easy to check that Condition 1 is naturally satisfied by Eq. (19). In addition, we can derive

$$
\begin{aligned}
& \frac{\partial H}{\partial u_{j-1 / 2}^{C}}=\frac{1}{4}\left[1+\lambda\left(f_{u}\right)_{j-1 / 2}^{C}\right]^{2}-\frac{\lambda^{2}}{2}\left(f_{u}\right)_{j-1 / 2}^{C}\left(f_{u u}\right)_{j-1 / 2}^{C}\left(u_{j-1 / 2}^{R}-u_{j-1 / 2}^{C}\right), \\
& \frac{\partial H}{\partial u_{j-1 / 2}^{R}}=\frac{1}{4}\left\{1-\left[\lambda\left(f_{u}\right)_{j-1 / 2}^{C}\right]^{2}\right\}, \\
& \frac{\partial H}{\partial u_{j+1 / 2}^{C}}=\frac{1}{4}\left[1-\lambda\left(f_{u}\right)_{j+1 / 2}^{C}\right]^{2}+\frac{\lambda^{2}}{2}\left(f_{u}\right)_{j+1 / 2}^{C}\left(f_{u u}\right)_{j+1 / 2}^{C}\left(u_{j+1 / 2}^{C}-u_{j+1 / 2}^{L}\right), \\
& \frac{\partial H}{\partial u_{j+1 / 2}^{L}}=\frac{1}{4}\left\{1-\left[\lambda\left(f_{u}\right)_{j+1 / 2}^{C}\right]^{2}\right\} .
\end{aligned}
$$

If the CFL condition [11]

$$
\lambda \max \left|f_{u}^{C}\right| \leq 1
$$

holds, a sufficient condition for satisfying Condition 2 expressed by Eq. (15) is

$$
\frac{1}{4}\left(1-\left|\lambda f_{u}^{C}\right|\right)^{2}-\frac{\lambda^{2}}{4}\left|f_{u}^{C} f_{u u}^{C} u_{x}\right| \Delta x \geq 0 \text { for all } j
$$

Solving this inequality gives us the following CFL-like condition

$$
\lambda \max \left(\left|f_{u}^{C}\right|+\sqrt{\left|f_{u}^{C} f_{u u}^{C} u_{x} \Delta x\right|}\right) \leq 1 .
$$

For linear problems $f_{u u}=0$, which implies the standard CFL condition $\lambda \max \left|f_{u}^{C}\right| \leq 1$, but for non-linear cases, the constraint of CFL condition is more restrictive. Note that, $\left|f_{u}^{C} f_{u u}^{C} u_{x} \Delta x\right|$ is $O(\Delta x)$ for continuous problems, so Eq. (23) will approach to Eq. (21) when $\Delta x$ approaches to 0 .

Since $u_{j-1 / 2}^{C}$ and $u_{j+1 / 2}^{C}$ are the values at solution points, $u_{j-1 / 2}^{C}, u_{j+1 / 2}^{C} \in[m, M]$ will be satisfied automatically for maximum-principle-satisfying CE/SE schemes. To guarantee $u_{j-1 / 2}^{R}, u_{j+1 / 2}^{L} \in[m, M]$, we employ the following limiter [1] for derivatives

$$
\left(u_{x}\right)_{j \pm 1 / 2}^{\text {new }}=\left(u_{x}\right)_{j \pm 1 / 2}^{C E S E} * \operatorname{MIN}\left(1, \theta_{1}, \theta_{2}\right),
$$

where

$$
\theta_{1}=2\left|\frac{M-u_{j \pm 1 / 2}}{\left(u_{x}\right)_{j \pm 1 / 2}^{C E S E} \Delta x}\right|, \theta_{2}=2\left|\frac{m-u_{j \pm 1 / 2}}{\left(u_{x}\right)_{j \pm 1 / 2}^{C E S E} \Delta x}\right| .
$$


Here, the superscripts ' $n e w$ ' and 'CESE' indicate the reconstructed and the calculated values using the $\mathrm{CE} / \mathrm{SE}$ scheme, respectively. This limiter was proposed by Zhang and Shu [1], who proved that this limiter preserves the accuracy of the original polynomial. Thus, the accuracy of the overall CE/SE discretization is also reserved. In addition, because the limiter only changes the value of the derivatives, the conservativeness of $\mathrm{CE} / \mathrm{SE}$ scheme is still guaranteed.

The implementation of the proposed approach is straightforward in an existing code because the whole procedure is local and only affects the reconstructed cell itself. Two things deserve to be highlighted. First, the limiting process used in Eq. (24) only plays the role of a secondary limiter to guarantee the maximum principle; the WBAP limiter is still required for shock capturing. And second, since central and upwind CE/SE schemes have the same updating scheme for mesh quantities, the proposed approach is applicative for both central and upwind CE/SE schemes.

\subsection{Two dimensional maximum-principle-satisfying CE/SE scheme}

The proof for the 2D case follows immediately by expanding all of the terms on the right hand side (RHS) of Eq. (12). Since the expanded equations are verbose, we put them in Appendix A. By inserting Eqs. (A.14) (A.25) into Eq. (12), one can obtain the general form of 2D CE/SE schemes on uniform structured mesh as

$$
\begin{aligned}
& u_{i, j}^{n}=H\left(u_{i-1 / 2, j-1 / 2}^{C}, u_{i-1 / 2, j-1 / 2}^{R}, u_{i-1 / 2, j-1 / 2}^{R U}, u_{i-1 / 2, j-1 / 2}^{U},\right. \\
& u_{i+1 / 2, j-1 / 2}^{C}, u_{i+1 / 2, j-1 / 2}^{U}, u_{i+1 / 2, j-1 / 2}^{L U}, u_{i+1 / 2, j-1 / 2}^{L}, \\
& u_{i+1 / 2, j+1 / 2}^{C}, u_{i+1 / 2, j+1 / 2}^{L}, u_{i+1 / 2, j+1 / 2}^{L D}, u_{i+1 / 2, j+1 / 2}^{D}, \\
& \left.u_{i-1 / 2, j+1 / 2}^{C}, u_{i-1 / 2, j+1 / 2}^{D}, u_{i-1 / 2, j+1 / 2}^{R D}, u_{i-1 / 2, j+1 / 2}^{R}\right) .
\end{aligned}
$$

It is not difficult to check that Condition 1 in Section 3.1 is satisfied by Eq. (26). Now we will put emphasis on Condition 2. First, we derive the partial difference of $H$ with respect to each term in the bracket of Eq. (26), which is presented in Appendix B. For linear cases, $f_{u u}=g_{u u}=0$ which implies that all non-linear terms in Eqs. (B.1) (B.16) disappear. If the 2D CFL condition

$$
\max \left(\lambda_{x}\left|f_{u}^{C}\right|+\lambda_{y}\left|g_{u}^{C}\right|\right) \leq 1
$$


holds, $\partial H / \partial q(q$ can be any term in the bracket of Eq. (26)) is obviously nonnegative. In other words, the maximum principle is satisfied by 2D CE/SE schemes for linear cases under the standard CFL condition Eq. (27). For non-linear cases, non-linear terms appear in Eqs. (B.1), (B.5), (B.9) and (B.13) which are very similar to each other. Condition 2 requires that

$$
G(\Delta t) \equiv\left[1-\mu_{0} \Delta t\right]^{2}-\mu_{1} \Delta t-\left(\mu_{2}+\mu_{3}\right) \Delta t^{2} \geq 0, \text { for all } i, j .
$$

where

$$
\begin{gathered}
\mu_{0}=\left|f_{u}^{C}\right| / \Delta x+\left|g_{u}^{C}\right| / \Delta y, \\
\mu_{1}=\left|f_{u u}^{C} u_{y}\right| \Delta y / \Delta x+\left|g_{u u}^{C} u_{x}\right| \Delta x / \Delta y, \\
\mu_{2}=\left(\left|f_{u u}^{C}\right| / \Delta x+\left|g_{u u}^{C}\right| / \Delta y\right)\left|f_{u}^{C} u_{x}+g_{u}^{C} u_{y}\right|, \\
\mu_{3}=\left(\left|f_{u}^{C}\right| / \Delta x+\left|g_{u}^{C}\right| / \Delta y\right)\left|f_{u u}^{C} u_{x}+g_{u u}^{C} u_{y}\right| .
\end{gathered}
$$

Obviously $\mu_{0}, \mu_{1}, \mu_{2}, \mu_{3} \geq 0$, it is easy to check that $G(\Delta t)$ is a monotonically decreasing function when $0 \leq \mu_{0} \Delta t \leq 1$. On the other hand, $G(0)=1>0$ and $G\left(1 / \mu_{0}\right)=-\left(\frac{\mu_{1}}{\mu_{0}}+\frac{\mu_{2}+\mu_{3}}{\mu_{0}^{2}}\right) \leq 0$. Therefore, we can seek one, and only one, solution $\Delta t_{0}$ such that Eq. (28) holds for $\Delta t \in\left[0, \Delta t_{0}\right]$. Solving $G(\Delta t)=0$, yields

$$
\Delta t_{0}=\frac{2}{2 \mu_{0}+\mu_{1} \pm \sqrt{\mu_{1}^{2}+4\left(\mu_{0} \mu_{1}+\mu_{2}+\mu_{3}\right)}} \text {. }
$$

Obviously, only

$$
\Delta t_{0}=\frac{2}{2 \mu_{0}+\mu_{1}+\sqrt{\mu_{1}^{2}+4\left(\mu_{0} \mu_{1}+\mu_{2}+\mu_{3}\right)}} \in\left[0,1 / \mu_{0}\right]
$$

which is the desired solution.

Therefore, if the CFL-like condition

$$
\Delta t \max \left[2 \mu_{0}+\mu_{1}+\sqrt{\mu_{1}^{2}+4\left(\mu_{0} \mu_{1}+\mu_{2}+\mu_{3}\right)}\right] \leq 2
$$

holds, the maximum principle can be satisfied by 2D CE/SE scheme. For linear case, $\mu_{1}=\mu_{2}=\mu_{3}=0$ and Eq. (31) will recover the standard CFL condition Eq. (27). 
What remains is to limit all the values in the bracket of Eq. (26) in the desired range of $[m, M]$. Thus, we have to guarantee $u \in[m, M]$ in every cell. Because a piecewise linear assumption is employed, the maximum or the minimum value in each cell can only appear at the four corners. This means that we only need to guarantee $u_{i, j}^{L D}, u_{i, j}^{R D}, u_{i, j}^{R U}$ and $u_{i, j}^{L U} \in[m, M]$. To achieve this goal, we define new coordinates, $\xi$ and $\eta$, which point the diagonal directions of the cell, as shown in Fig. 4. Using coordinate transformation, we have

$$
\begin{aligned}
& u_{\xi}^{C E S E}=u_{x}^{C E S E} \frac{\partial x}{\partial \xi}+u_{y}^{C E S E} \frac{\partial y}{\partial \xi} \\
& u_{\eta}^{C E S E}=u_{x}^{C E S E} \frac{\partial x}{\partial \eta}+u_{y}^{C E S E} \frac{\partial y}{\partial \eta}
\end{aligned}
$$

where

$$
\left[\begin{array}{ll}
\frac{\partial x}{\partial \xi} & \frac{\partial y}{\partial \xi} \\
\frac{\partial x}{\partial \eta} & \frac{\partial y}{\partial \eta}
\end{array}\right]=\left[\begin{array}{cc}
\cos \theta & \sin \theta \\
-\cos \theta & \sin \theta
\end{array}\right]
$$

Following the idea of the 1D scheme, we employ the slope limiter

$$
\left(u_{X}\right)_{i, j}^{\text {new }}=\left(u_{X}\right)_{i, j}^{C E S E} * \operatorname{MIN}\left(1, \theta_{1}, \theta_{2}\right)
$$

where,

$$
\theta_{1}=2\left|\frac{M-u_{i, j}^{C}}{u_{X}^{C E S E} \sqrt{\Delta x^{2}+\Delta y^{2}}}\right|, \theta_{2}=2\left|\frac{m-u_{i, j}^{C}}{u_{X}^{C E S E} \sqrt{\Delta x^{2}+\Delta y^{2}}}\right| .
$$

Here $X$ represents $\xi$ or $\eta$. After applying this limiter, the value of $u$ can be confined in the range of $[m, M]$ and it will not change the cell average. After the limiting procedure, the inverse operation of Eq. (32) can be used to get the derivatives with respect to $x$ and $y$ for the next time step.

\section{Extension to reduced five-equation model for compressible multifluids}

\subsection{Physical model}

Compressible multifluid flows are very common in nature $[25,26]$ and relevant simulations have attracted considerable interest. Generally speaking, there are two-types of compressible multifluid models. The first one is the sharp interface 
model in which the interface between different fluids is treated as a sharp discontinuity. Usually, the volume of fluid (VOF) method [27], the level set method [28-30] or the Lagrangian front tracking method [31,32] is employed to trace the precise position of the interface, and ghost fluid method is used to treat the interfacial boundary condition. Alternatively, the diffusive interface model does not require the precise location of the interface. Instead, each phase is marked by a color function, which, because of numerical diffusion, can take intermediate values between 0 and 1 . The Baer-Nunziato model [33] is a classical diffusive interface model consisting of seven equations (two mass conservation equations, two momentum equations, two energy (or pressure) equations and one topological equation (color function)) for 1D two-fluid flows. This model has several advantages: it is unconditionally hyperbolic, it is able to treat multiphase mixtures as well as interface problems between pure fluids and it allows the treatment of fluids characterized by very different thermodynamics because each fluid uses its own equation of state. However, these models are numerically complex to solve because of the large number of waves they contain and because of the sensibility of the results with respect to the relaxation procedures. The five-equation model is a reduced model for compressible two phase flows. In this type of model, the velocity and pressure (or temperature) of different components are considered in equilibrium (See, for example, [4-8] and [34-36]). It provides an interesting alternative to the seven-equation model because it is cheaper, simpler to implement and easily extensible to an arbitrary number of materials [36].

Saurel and Abgrall $[4,34]$ proposed that in any flow where the pressure and the velocity are uniform, pressure and velocity fields should remain uniform at all times. Based on this physical reality, the volume fraction-based five-equation model can be expressed as [6] 


$$
\begin{aligned}
& \frac{\partial \alpha_{i}}{\partial t}+\mathbf{V} \cdot \nabla \alpha_{i}=0, i=1 \text { or } 2 \\
& \frac{\partial \rho_{s} \alpha_{s}}{\partial t}+\nabla \cdot\left(\rho_{s} \alpha_{s} \mathbf{V}\right)=0, s=1 \sim 2 \\
& \frac{\partial \rho \mathbf{V}}{\partial t}+\nabla \cdot(\rho \mathbf{V} \otimes \mathbf{V}+p)=0 \\
& \frac{\partial E}{\partial t}+\nabla \cdot(\mathbf{V}(E+p))=0
\end{aligned}
$$

where $\alpha_{i}$ denotes the volume fraction of fluid $i, \rho_{s}$ the density of fluid $s, \rho$ the density of the mixture, $\mathbf{V}$ the velocity vector, $p$ the pressure and $E$ the total energy.

A specific equation of state (EOS) is required to close the system. Typical choices for the EOS, for example, are the ideal gas [4], the van der Waals gas [7], the stiffened gas $[6,34]$ and the Mie-Grüneisen [8]. Here, we adopt the stiffened gas EOS given the following relationship:

$$
p=(\gamma-1)\left(E-\frac{1}{2} \rho \mathbf{V} \cdot \mathbf{V}\right)-\gamma \pi
$$

where,

$$
\frac{1}{\gamma-1}=\sum \frac{\alpha_{i}}{\gamma_{i}-1} \text { and } \frac{\gamma \pi}{\gamma-1}=\sum \frac{\alpha_{i} \gamma_{i} \pi_{i}}{\gamma_{i}-1} .
$$

The total density and the sound speed of the mixture can be respectively calculated as

$$
\rho=\sum \alpha_{i} \rho_{i} \text { and } c=\sqrt{\gamma(p+\pi) / \rho} .
$$

Note that, this model can prevent pressure oscillation across the contact surface, but a further treatment is required to prevent temperature oscillations [37].

\subsection{Algorithm implementation}

The five-equation model is a quasi-conservative approach for compressible multifluid flows. To solve this model accurately, the basic requirements for the numerical algorithm are conservativeness and shock- and interface-capturing capabilities. In addition, positivity preserving for the volume fractions is also extremely important, especially for multifluid flows with large density ratios. The central $\mathrm{CE} / \mathrm{SE}$ scheme is conservative and can capture shocks very well, but it is extremely diffusive to capture interface (See [18]). The upwind CE/SE scheme 
inherits the properties of the central scheme and significantly improves the capacity to capture the interface. Furthermore, it satisfies the maximum-principle by adopting the simple limiter expressed by Eq. (24) and (34). (, which are also applicable for central $\mathrm{CE} / \mathrm{SE}$ schemes). For these reasons, the maximum-principle satisfying upwind CE/SE scheme is used herein to solve the five-equation model.

When applying upwind CE/SE schemes to solve the Euler equation, a local Riemann solver is required to get $F_{C}, F_{C D}, F_{C U}, G_{C L}$ and $G_{C R}$ in Eqs. (7) (11). In previous studies [16, 17], we compared the computational performance of upwind CE/SE schemes, coupled with HLL, HLLC and Roe's Riemann solvers, for single-fluid flows. We showed that the HLLC-CE/SE solver efficiently captures the contact discontinuity with relatively low computational cost. Moreover, the HLLC Riemann solver has positivity-preserving properties for scalar quantity and is widely used in simulations of compressible multifluid flows (see, for example, [38-41]). For these reasons, in this work, we solve the five-equation model for multifluid flows using a HLLC-CE/SE solver. The specific formula for the HLLC Riemann solver can be found in Refs. [38-40] and details of the HLLC-CE/SE solver can be found in Refs. [16] and [17].

The procedure of the algorithm can be described as follows:

(1) Use the HLLC-CE/SE solver to update the density of each fluid $\left(\rho_{s} \alpha_{s}\right)$, the momentum $(\rho \mathbf{V})$ and the total energy $(E)$.

(2) Use the updated velocity derived by the CE/SE solver as the transporting velocity of the advection equation for the volume fraction (i.e. the first equation of Eq. (36)), and update either the volume fraction using the upwind CE/SE scheme (Eqs. (6) and (7) for $1 \mathrm{D}$ and Eqs. (8) (12) for 2D). This procedure leads to the oscillation-free advection of an isolated interface and propagation of isolated shocks [38].

(3) Get the parameters $\theta_{1}$ and $\theta_{2}$ in the limiter by inserting the updated volume fraction and its spatial derivatives into Eq. (25) or (35). For 2D cases, the derivatives must be first transformed to $\xi$ and $\eta$ directions using Eq. (32).

(4) Implement the limiter, expressed by (24) or (34), not only for the derivatives of the volume fraction, but also for the derivatives of other conserved quantities. Note 
that, we must use the same $\theta_{1}$ and $\theta_{2}$ derived in step (3) for all quantities. This is to guarantee consistency between computations of volume fractions and the energy equation which is crucial for oscillation-free advection of an isolated interface. For 2D cases, the derivatives must be transformed back to $x$ and $y$ directions using the inverse operation of Eq. (36).

(5) Repeat the procedure.

As demonstrated in Section 3, after implementing the limiter, CE/SE schemes satisfy the under an available CFL-like condition. If $\mathbf{F}(u)$ in Eq. (1) is linear with respect to $u$, maximum-principle-satisfying $\mathrm{CE} / \mathrm{SE}$ schemes have the loosest $\mathrm{CFL}$ condition. In step 2 the transporting velocity in each CE is constant. In other words, the advection equation for the volume fraction in Eq. (41) is linear with respect to $\alpha_{i}$. Therefore, the maximum-principle-satisfying CE/SE scheme for solving the five-equation model requires a standard CFL condition. For the non-linear cases, the reinforced CFL condition (Eq. (23) for 1D and Eq. (31) for 2D) is used.

\section{Numerical examples}

Example 1. Modelling rarefaction with a stationary shock using inviscid Burgers' equation

We consider the 1D inviscid Burgers' equation

$$
\frac{\partial u}{\partial t}+\frac{\partial u^{2} / 2}{\partial x}=0
$$

with the following initial condition $[42,43]$

$$
u=\left\{\begin{array}{l}
1,|x|<1 / 3, \\
-1,1 / 3 \leq|x| \leq 1 .
\end{array}\right.
$$

This case is used to test the maximum-principle satisfying CE/SE scheme for non-linear cases. The simulation is run up to $t=0.32$ using 200 grid-points. Fig. 5 shows the comparison of the results computed by the CE/SE scheme without and with the limiter. Hereafter, 'limiter' means the secondary limiter expressed by Eq. (24) or Eq. (34). We observed that the proposed scheme can effectively capture the stationary shock and rarefaction. Although in the absence of the limiter, overshot takes place 
near the shock, once the limiter is applied, these overshoots are tamed and the value of $u$ never jumps out the initial range of $[-1,1]$.

\section{Example 2. Isolated interface evolution in one spatial dimension}

This case is used to test the properties of capturing interfaces, conservativeness and satisfaction of the maximum principle of our scheme. The computational domain is $[0$, 2]. Two configurations are considered here. The first is a gas-gas interface evolution with the initial condition described as

$$
(\rho, u, p, \gamma, \pi)=\left\{\begin{array}{l}
(10,1,1,1.667,0), 0.5<x \leq 1.5 \\
(1,1,1,1.4,0), \text { else }
\end{array}\right.
$$

The second is a gas-water interface evolution with the initial condition

$$
(\rho, u, p, \gamma, \pi)=\left\{\begin{array}{l}
\left(1000,100,10^{5}, 4.4,6 \times 10^{8}\right), 0.5<x \leq 1.5 \\
\left(1,100,10^{5}, 1.4,0\right), \text { else }
\end{array}\right.
$$

Periodic boundary conditions are applied to both sides of the domain. The first and the second configurations are computed to $t=2$ and $t=0.02$ with 200 grid points, which means the interface propagates one period and should return to its original position. Fig. 6 shows the density profiles, the errors of velocity and pressure, the errors of conserved quantities and the histories of minimum volume fractions for the first configuration computed without (left column) and with (right column) the limiter. The error of $X$ is calculated as

$$
\operatorname{Error}(X)=\frac{X-X_{0}}{X_{0}},
$$

where $X_{0}$ denotes the theoretical value of $X$. It is observed that the present scheme is conservative and captures the interface accurately without spurious oscillations. The scheme with the limiter strictly maintains positive volume fractions, but without the limiter, minimum volume fractions become negative. Without the limiter, simulation the gas-water interface propagation will diverge. Fig. 7 illustrates these results calculated by the scheme with limiter which are similar to those of gas-gas propagation.

\section{Example 3. The gas-liquid Riemann problem}


This is a shock-tube problem used to model underwater explosions [6, 38]. The initial condition is defined as

$$
(\rho, u, p, \gamma, \pi)=\left\{\begin{array}{l}
(1.241,0,2.753,1.4,0),-1 \leq x \leq 0, \\
\left(0.991,0,3.059 \times 10^{-4}, 5.5,1.505\right), 0<x \leq 1 .
\end{array}\right.
$$

The large pressure ratio makes this a challenging numerical simulation. We use the maximum-principle-satisfying CE/SE scheme to simulate this problem with 200 grid points. Fig. 8 shows the density, velocity, pressure and $\gamma$ profiles at $t=0.2$. The proposed scheme shows good shock and interface capturing capabilities. In addition, Fig. 9 shows that the scheme is conservative and preserves the positivity of the volume fractions.

Example 4. Richtmyer-Meshkov instabilities developing from a circular interface This example has been carried out in [36] and [44] to assess the robustness of the numerical methods for the 2D computation of multifluid flows. The computational domain is $[0,0.8] \times[0,0.8]$ with the initial condition set as

$$
(\rho, u, v, p, \gamma, \pi)=\left\{\begin{array}{l}
\left(10,0,0,10^{6}, 3,0\right), \text { if } 0.55 \leq R \leq 0.6 \\
\left(1,0,0,10^{5}, 1.4,0\right), \text { else }
\end{array}\right.
$$

where $R=\sqrt{(x-0.8)^{2}+y^{2}}$. Fig. 10 (a) shows the initial configuration, where the heavy gas sits in the black zone. A symmetric boundary condition is applied to the right and lower sides, and a non-reflection boundary condition is applied to the left and upper sides. The simulation is first performed using $400 \times 400$ grid points, which is consistent with that used in [36], but less than that used in [44]. Some perturbations in the order of the mesh size will be generated due to the use of a rectangular mesh to approach circular surfaces. In addition, the propagating shocks, which are initiated from the pressure jump, will accelerate the interfaces in the normal direction. As a result, numerous Richtmyer-Meshkov instabilities develop near the interfaces which are well captured, as shown in Fig. 10. Moreover, the mass of the heavy gas is conserved to truncation errors of a computer and the volume fractions are positive throughout the simulation, as shown in Fig. 11. To evaluate the computational costs of the secondary limiter, we run this example using different grid points with and 
without the secondary limiter. The code was parallelized using OpenMP. We tested the computational costs on a laptop equipped with 8 Intel ${ }^{\circledR}$ Core $^{\mathrm{TM}}$ i7-4720HQ CPUs @ 2.60GHz. Table 1 presents the corresponding computational time. It is observed that the secondary limiter increases computational costs of about $2 \%$ which is relatively small.

\section{Example 5. Shock-bubble interaction}

This is a classic test case for a solver of compressible multifluids solver (See, for example, [18, 28-30, 32 and 38]). The initial setup of the computation is shown in Fig. 12. Symmetry is forced on the lower boundary such that we only simulate half of the physical domain. The nonreflecting boundary condition is used on the left and right sides and the reflective boundary condition is applied to the upper boundary. The radius of the bubble is $R=25 \mathrm{~mm}$. Other geometric dimensions are set as $W=1.78 R$, $L_{1}=2 R, L_{2}=8 R$ and $D_{s}=0.2 R$. The initial condition is

$$
(\rho, u, v, p, \gamma, \pi)=\left\{\begin{array}{l}
(1.29,0,0,101325,1.4,0), \text { pre-shocked air } \\
(0.214,0,0,101325,5 / 3,0), \text { inside the bubble }
\end{array}\right.
$$

The status of post-shocked air is calculated according to the normal shock relationship. The Mach number of the shock is chosen as 1.22 which is consistent with the literature. The simulation is performed using $1600 \times 400$ mesh grids. After the simulation begins, the planar shock propagates from left to right and impacts the bubble. Because there is a mismatch of acoustic impedance between the bubble and the air, reflection and refraction of the shock occur at the interface. In addition, some anticlockwise vorticities generate near the interface due to the baroclinicity $\nabla \rho \times \nabla p$. More details about shock-bubble dynamics can be found in [25]. Fig. 13 shows the morphology of the bubble at selected times. The interface is captured clearly and the corresponding phenomena are comparable with previous studies. Compared with results computed by the central CE/SE scheme in [18], the interface is distinctly sharper. In [32], Terashima and Tryggvason compared the precise positions of the upstream point, the jet point and the downstream point of the bubble derived by the front tracking and the level set methods; results were similar. To quantitatively 
validate the present scheme, we compare our results with those derived by the level set method in Fig. 14, which shows that the trajectories of different positions are a good match with those in the literature. Fig. 15, shows that the mass of the bubble is conserved and that the minimum volume fractions remain positive throughout the simulation.

\section{Example 6. Shock-water-column interaction}

This example has been performed several times before (See, for example, [32, 39, 41, 45 and 46]). The initial setup of this problem is similar with that of the shock-bubble interaction problem (See Fig. 12), but beyond that, this problem becomes considerably more difficult due to the high density ratio and the large difference between the thermodynamic properties of air and water. The non-reflecting boundary condition is applied to the upper side, and the other boundary conditions are the same as those in Example 5. The radius of the water droplet is $R=2.4 \mathrm{~mm}$. Other geometric dimensions are set as $W=10 R, L_{1}=-15 R, L_{2}=25 R$ and $D_{s}=0.2 R$. The initial condition is

$$
(\rho, u, v, p, \gamma, \pi)=\left\{\begin{array}{l}
(1.225,0,0,101325,1.4,0), \text { pre-shocked air, } \\
\left(1000,0,0,101325,4.4,6 \times 10^{8}\right) \text {, inside the bubble. }
\end{array}\right.
$$

The status of post-shocked air is calculated according to the normal shock relation. A case with the Mach number of the shock (Ms) equal to 1.47 is computed using $2400 \times 600$ mesh points. Here, completely different phenomena are observed in comparison with the shock-bubble interaction problem. Because the density ratio is really high, the deformation of the water column becomes invisible at an early stage; Fig. 16 shows the sequences of pressure contours at this early stage. The shock propagates through the water column, just like passing over a rigid cylinder. Because the pressure wave propagates much faster in water than in air, the large difference of acoustic impedance causes the wave to become trapped in the water column and bounce back and forth. As a result, the pressure inside the water column changes very quickly and is highly heterogeneous in preparation for the upcoming deformation. In Fig. 17, we show the stripping breakup process of the water column presented by an optimized numerical Schlieren plot, which is generated by the function 
$\left(1+\alpha_{\text {air }}^{2}\right) \log (|\nabla \rho|+1)$ [45]. After the incident shock passes the water column, regular reflection first occurs at the symmetric boundary (See Fig. 16 (f)) and then it will transform to Mach reflection (See Fig. 17 (a)). Meanwhile, large recirculation regions form behind the column, eventually flatting the column [32, 41]. Ultimately, the column is stripped away due to the large difference between the velocity of air and that of water. In general, the phenomena we observed agree with the results of [41], but we also recognized that the instabilities are more detailed in our simulation.

For quantitative validation, we trace the position of the center of the water column and compare the drag coefficient with Chen's simulation [41] and Igra's experimental data [47]. The drag coefficient is defined as $C d=\frac{F}{0.5 \rho_{g} u_{g}^{2} D}$, where $F, \rho_{g}, u_{g}$ and $D$ are the drag force, density of freestream, velocity of freestream and the diameter of the water column, respectively. As seen in Fig. 18, where time is normalized as $t^{*}=t u_{g} / D$, good agreement is achieved. This case demonstrates the capability of the present scheme to handle a large density ratio.

\section{Conclusion}

A second-order maximum-principle-satisfying CE/SE scheme is constructed by adopting a very simple limiter which can preserve the conservativeness and accuracy of the scheme. For linear problems, the standard CFL condition is sufficient. For non-linear problems, a more restrictive CFL condition is required. Extensive numerical examples show that the proposed scheme is very robust and accurate in computing compressible multifluid flows even for the cases with a large density ratio and very different thermodynamic properties. Our scheme simultaneously captures shocks and contact surfaces sharply without spurious oscillations, while strictly preserving the conservation law and the positivity of volume fractions. Due to the special time marching strategy associated with CE/SE schemes, high-order maximum-principle-satisfying CE/SE schemes are complex and need further research.

\section{Acknowledgements}


H. Shen and C. Y. Wen were supported by NSFC grant 11372265 and the opening project of the State Key Laboratory of Explosion Science and Technology (Beijing Institute of Technology) grant KFJJ15-09M. C.-W. Shu was supported by ARO grant W911NF-15-1-0226 and NSF grant DMS-1418750. For computer time, this research used the resources of the Extreme Computing Research Center at King Abdullah University of Science \& Technology (KAUST) in Thuwal, Saudi Arabia.

\section{Appendix A. Expanded terms of 2D CE/SE scheme}

Using the Taylor expansion in $\mathrm{SE}_{i-1 / 2, j-1 / 2}^{n-1 / 2}, \mathrm{SE}_{i+1 / 2, j-1 / 2}^{n-1 / 2}, \mathrm{SE}_{i+1 / 2, j+1 / 2}^{n-1 / 2}$ and $\mathrm{SE}_{i-1 / 2, j+1 / 2}^{n-1 / 2}$, the fluxes in Eq. (12) can be calculated as

$$
\begin{aligned}
& U_{L D}=u_{i-1 / 2, j-1 / 2}+\left(u_{x}\right)_{i-1 / 2, j-1 / 2} \frac{\Delta x}{4}+\left(u_{y}\right)_{i-1 / 2, j-1 / 2} \frac{\Delta y}{4}, \\
& F_{L D}=f_{i-1 / 2, j-1 / 2}+\left(f_{y}\right)_{i-1 / 2, j-1 / 2} \frac{\Delta y}{4}+\left(f_{t}\right)_{i-1 / 2, j-1 / 2} \frac{\Delta t}{4}, \\
& G_{D L}=g_{i-1 / 2, j-1 / 2}+\left(g_{x}\right)_{i-1 / 2, j-1 / 2} \frac{\Delta x}{4}+\left(g_{t}\right)_{i-1 / 2, j-1 / 2} \frac{\Delta t}{4}, \\
& U_{R D}=u_{i+1 / 2, j-1 / 2}-\left(u_{x}\right)_{i+1 / 2, j-1 / 2} \frac{\Delta x}{4}+\left(u_{y}\right)_{i+1 / 2, j-1 / 2} \frac{\Delta y}{4}, \\
& F_{R D}=f_{i+1 / 2, j-1 / 2}+\left(f_{y}\right)_{i+1 / 2, j-1 / 2} \frac{\Delta y}{4}+\left(f_{t}\right)_{i+1 / 2, j-1 / 2} \frac{\Delta t}{4}, \\
& G_{D R}=g_{i+1 / 2, j-1 / 2}-\left(g_{x}\right)_{i+1 / 2, j-1 / 2} \frac{\Delta x}{4}+\left(g_{t}\right)_{i+1 / 2, j-1 / 2} \frac{\Delta t}{4}, \\
& U_{R U}=u_{i+1 / 2, j+1 / 2}-\left(u_{x}\right)_{i+1 / 2, j+1 / 2} \frac{\Delta x}{4}-\left(u_{y}\right)_{i+1 / 2, j+1 / 2} \frac{\Delta y}{4}, \\
& F_{R U}=f_{i+1 / 2, j+1 / 2}-\left(f_{y}\right)_{i+1 / 2, j+1 / 2} \frac{\Delta y}{4}+\left(f_{t}\right)_{i+1 / 2, j+1 / 2} \frac{\Delta t}{4}, \\
& G_{U R}=g_{i+1 / 2, j+1 / 2}-\left(g_{x}\right)_{i+1 / 2, j+1 / 2} \frac{\Delta x}{4}+\left(g_{t}\right)_{i+1 / 2, j+1 / 2} \frac{\Delta t}{4}, \\
& U_{L U}=u_{i-1 / 2, j+1 / 2}+\left(u_{x}\right)_{i-1 / 2, j+1 / 2} \frac{\Delta x}{4}-\left(u_{y}\right)_{i-1 / 2, j+1 / 2} \frac{\Delta y}{4}, \\
& F_{L U}=f_{i-1 / 2, j+1 / 2}-\left(f_{y}\right)_{i-1 / 2, j+1 / 2} \frac{\Delta y}{4}+\left(f_{t}\right)_{i-1 / 2, j+1 / 2} \frac{\Delta t}{4}, \\
& G_{U L}=g_{i-1 / 2, j+1 / 2}+\left(g_{x}\right)_{i-1 / 2, j+1 / 2} \frac{\Delta x}{4}+\left(g_{t}\right)_{i-1 / 2, j+1 / 2} \frac{\Delta t}{4} .
\end{aligned}
$$

Note that, the superscript ' $n-1 / 2$ ' is dropped for convenience. Following the abbreviations in 1D scheme, we use superscripts $C, L, R, D$ and $U$ to denote values at the center and the left, the right, the down, and the upper side of an element (see Fig. 
3 for clarity). If we define $\delta_{i j}^{A, B}=u_{i j}^{A}-u_{i j}^{B}$, then the spatial derives of $u$ can be written as

$$
\begin{aligned}
& \left(u_{x}\right)_{i, j} \frac{\Delta x}{2}=\delta_{i, j}^{R, C}=\delta_{i, j}^{C, L}=\delta_{i, j}^{R U, U}=\delta_{i, j}^{U, L U}=\delta_{i, j}^{R D, D}=\delta_{i, j}^{D, L D}, \\
& \left(u_{y}\right)_{i, j} \frac{\Delta y}{2}=\delta_{i, j}^{U, C}=\delta_{i, j}^{C, D}=\delta_{i, j}^{R U, R}=\delta_{i, j}^{R, R D}=\delta_{i, j}^{L U, L}=\delta_{i, j}^{L, L D} .
\end{aligned}
$$

Plugging Eq. (A.13) into Eqs. (A.1) (A.12), one derives

$$
\begin{aligned}
& U_{L D}=\frac{1}{4}\left(u_{i-1 / 2, j-1 / 2}^{C}+u_{i-1 / 2, j-1 / 2}^{R}+u_{i-1 / 2, j-1 / 2}^{R U}+u_{i-1 / 2, j-1 / 2}^{U}\right), \\
& F_{L D}=f_{i-1 / 2, j-1 / 2}^{C}+\frac{1}{2}\left(f_{u}\right)_{i-1 / 2, j-1 / 2}^{C} \delta_{i-1 / 2, j-1 / 2}^{U, C} \\
& -\frac{1}{4}\left(f_{u}\right)_{i-1 / 2, j-1 / 2}^{C}\left[\lambda_{x}\left(f_{u}\right)_{i-1 / 2, j-1 / 2}^{C}\left(\delta_{i-1 / 2, j-1 / 2}^{R U, U}+\delta_{i-1 / 2, j-1 / 2}^{R, C}\right)\right. \\
& \left.+\lambda_{y}\left(g_{u}\right)_{i-1 / 2, j-1 / 2}^{C}\left(\delta_{i-1 / 2, j-1 / 2}^{R U, R}+\delta_{i-1 / 2, j-1 / 2}^{U, C}\right)\right], \\
& G_{D L}=g_{i-1 / 2, j-1 / 2}^{C}+\frac{1}{2}\left(g_{u}\right)_{i-1 / 2, j-1 / 2}^{C} \delta_{i-1 / 2, j-1 / 2}^{R, C} \\
& -\frac{1}{4}\left(g_{u}\right)_{i-1 / 2, j-1 / 2}^{C}\left[\lambda_{x}\left(f_{u}\right)_{i-1 / 2, j-1 / 2}^{C}\left(\delta_{i-1 / 2, j-1 / 2}^{R U, U}+\delta_{i-1 / 2, j-1 / 2}^{R, C}\right)\right. \\
& \left.+\lambda_{y}\left(g_{u}\right)_{i-1 / 2, j-1 / 2}^{C}\left(\delta_{i-1 / 2, j-1 / 2}^{R U, R}+\delta_{i-1 / 2, j-1 / 2}^{U, C}\right)\right], \\
& U_{R D}=\frac{1}{4}\left(u_{i+1 / 2, j-1 / 2}^{C}+u_{i+1 / 2, j-1 / 2}^{U}+u_{i+1 / 2, j-1 / 2}^{L U}+u_{i+1 / 2, j-1 / 2}^{L}\right), \\
& F_{R D}=f_{i+1 / 2, j-1 / 2}^{C}+\frac{1}{2}\left(f_{u}\right)_{i+1 / 2, j-1 / 2}^{C} \delta_{i+1 / 2, j-1 / 2}^{U, C} \\
& -\frac{1}{4}\left(f_{u}\right)_{i+1 / 2, j-1 / 2}^{C}\left[\lambda_{x}\left(f_{u}\right)_{i+1 / 2, j-1 / 2}^{C}\left(\delta_{i+1 / 2, j-1 / 2}^{U, L U}+\delta_{i+1 / 2, j-1 / 2}^{C, L}\right)\right. \\
& \left.+\lambda_{y}\left(g_{u}\right)_{i+1 / 2, j-1 / 2}^{C}\left(\delta_{i+1 / 2, j-1 / 2}^{L U, L}+\delta_{i+1 / 2, j-1 / 2}^{U, C}\right)\right], \\
& G_{D R}=g_{i+1 / 2, j-1 / 2}^{C}-\frac{1}{2}\left(g_{u}\right)_{i+1 / 2, j-1 / 2}^{C} \delta_{i+1 / 2, j-1 / 2}^{C, L} \\
& -\frac{1}{4}\left(g_{u}\right)_{i+1 / 2, j-1 / 2}^{C}\left[\lambda_{x}\left(f_{u}\right)_{i+1 / 2, j-1 / 2}^{C}\left(\delta_{i+1 / 2, j-1 / 2}^{U, L U}+\delta_{i+1 / 2, j-1 / 2}^{C, L}\right)\right. \\
& \left.+\lambda_{y}\left(g_{u}\right)_{i+1 / 2, j-1 / 2}^{C}\left(\delta_{i+1 / 2, j-1 / 2}^{L U, L}+\delta_{i+1 / 2, j-1 / 2}^{U, C}\right)\right], \\
& U_{R U}=\frac{1}{4}\left(u_{i+1 / 2, j+1 / 2}^{C}+u_{i+1 / 2, j+1 / 2}^{L}+u_{i+1 / 2, j+1 / 2}^{L D}+u_{i+1 / 2, j+1 / 2}^{D}\right) \text {, }
\end{aligned}
$$




$$
\begin{aligned}
& F_{R U}=f_{i+1 / 2, j+1 / 2}^{C}-\frac{1}{2}\left(f_{u}\right)_{i+1 / 2, j+1 / 2}^{C} \delta_{i+1 / 2, j+1 / 2}^{C, D} \\
& -\frac{1}{4}\left(f_{u}\right)_{i+1 / 2, j+1 / 2}^{C}\left[\lambda_{x}\left(f_{u}\right)_{i+1 / 2, j+1 / 2}^{C}\left(\delta_{i+1 / 2, j+1 / 2}^{C, L}+\delta_{i+1 / 2, j+1 / 2}^{D, L D}\right)\right. \\
& \left.+\lambda_{y}\left(g_{u}\right)_{i+1 / 2, j+1 / 2}^{C}\left(\delta_{i+1 / 2, j+1 / 2}^{C, D}+\delta_{i+1 / 2, j+1 / 2}^{L, L D}\right)\right], \\
& G_{U R}=g_{i+1 / 2, j+1 / 2}^{C}-\frac{1}{2}\left(g_{u}\right)_{i+1 / 2, j+1 / 2}^{C} \delta_{i+1 / 2, j+1 / 2}^{C, L} \\
& -\frac{1}{4}\left(g_{u}\right)_{i+1 / 2, j+1 / 2}^{C}\left[\lambda_{x}\left(f_{u}\right)_{i+1 / 2, j+1 / 2}^{C}\left(\delta_{i+1 / 2, j+1 / 2}^{C, L}+\delta_{i+1 / 2, j+1 / 2}^{D, L D}\right)\right. \\
& \left.+\lambda_{y}\left(g_{u}\right)_{i+1 / 2, j+1 / 2}^{C}\left(\delta_{i+1 / 2, j+1 / 2}^{C, D}+\delta_{i+1 / 2, j+1 / 2}^{L, L D}\right)\right], \\
& U_{L U}=\frac{1}{4}\left(u_{i-1 / 2, j+1 / 2}^{C}+u_{i-1 / 2, j+1 / 2}^{D}+u_{i-1 / 2, j+1 / 2}^{R D}+u_{i-1 / 2, j+1 / 2}^{R}\right) \text {, } \\
& F_{L U}=f_{i-1 / 2, j+1 / 2}^{C}-\frac{1}{2}\left(f_{u}\right)_{i-1 / 2, j+1 / 2}^{C} \delta_{i-1 / 2, j+1 / 2}^{C, D} \\
& -\frac{1}{4}\left(f_{u}\right)_{i-1 / 2, j+1 / 2}^{C}\left[\lambda_{x}\left(f_{u}\right)_{i-1 / 2, j+1 / 2}^{C}\left(\delta_{i-1 / 2, j+1 / 2}^{R, C}+\delta_{i-1 / 2, j+1 / 2}^{R D, D}\right)\right. \\
& \left.+\lambda_{y}\left(g_{u}\right)_{i-1 / 2, j+1 / 2}^{C}\left(\delta_{i-1 / 2, j+1 / 2}^{C, D}+\delta_{i-1 / 2, j+1 / 2}^{R, R D}\right)\right], \\
& G_{U L}=g_{i-1 / 2, j+1 / 2}^{C}+\frac{1}{2}\left(g_{u}\right)_{i-1 / 2, j+1 / 2}^{C} \delta_{i-1 / 2, j+1 / 2}^{R, C} \\
& -\frac{1}{4}\left(g_{u}\right)_{i-1 / 2, j+1 / 2}^{C}\left[\lambda_{x}\left(f_{u}\right)_{i-1 / 2, j+1 / 2}^{C}\left(\delta_{i-1 / 2, j+1 / 2}^{R, C}+\delta_{i-1 / 2, j+1 / 2}^{R D, D}\right)\right. \\
& \left.+\lambda_{y}\left(g_{u}\right)_{i-1 / 2, j+1 / 2}^{C}\left(\delta_{i-1 / 2, j+1 / 2}^{C, D}+\delta_{i-1 / 2, j+1 / 2}^{R, R D}\right)\right] .
\end{aligned}
$$

where $f_{u}=\partial f / \partial u, g_{u}=\partial g / \partial u, \lambda_{x}=\Delta t / \Delta x$ and $\lambda_{y}=\Delta t / \Delta y$.

\section{Appendix B. Partial difference of Eq. (26)}

$$
\begin{aligned}
& \frac{\partial H}{\partial u_{i-1 / 2, j-1 / 2}^{C}}=\frac{1}{4} \frac{\partial U_{L D}}{\partial u_{i-1 / 2, j-1 / 2}^{C}}+\frac{\lambda_{x}}{4} \frac{\partial F_{L D}}{\partial u_{i-1 / 2, j-1 / 2}^{C}}+\frac{\lambda_{y}}{4} \frac{\partial G_{D L}}{\partial u_{i-1 / 2, j-1 / 2}^{C}} \\
& =\frac{1}{16}\left[1+\lambda_{x}\left(f_{u}\right)_{i-1 / 2, j-1 / 2}^{C}+\lambda_{y}\left(g_{u}\right)_{i-1 / 2, j-1 / 2}^{C}\right]^{2} \\
& +\frac{\lambda_{x}}{8}\left(f_{u u}\right)_{i-1 / 2, j-1 / 2}^{C} \delta_{i-1 / 2, j-1 / 2}^{U, C}+\frac{\lambda_{y}}{8}\left(g_{u u}\right)_{i-1 / 2, j-1 / 2}^{C} \delta_{i-1 / 2, j-1 / 2}^{R, C} \\
& -\frac{1}{8}\left[\lambda_{x}\left(f_{u u}\right)_{i-1 / 2, j-1 / 2}^{C}+\lambda_{y}\left(g_{u u}\right)_{i-1 / 2, j-1 / 2}^{C}\right] \\
& \times\left[\lambda_{x}\left(f_{u}\right)_{i-1 / 2, j-1 / 2}^{C} \delta_{i-1 / 2, j-1 / 2}^{R, C}+\lambda_{y}\left(g_{u}\right)_{i-1 / 2, j-1 / 2}^{C} \delta_{i-1 / 2, j-1 / 2}^{U, C}\right] \\
& -\frac{1}{8}\left[\lambda_{x}\left(f_{u}\right)_{i-1 / 2, j-1 / 2}^{C}+\lambda_{y}\left(g_{u}\right)_{i-1 / 2, j-1 / 2}^{C}\right] \\
& \times\left[\lambda_{x}\left(f_{u u}\right)_{i-1 / 2, j-1 / 2}^{C} \delta_{i-1 / 2, j-1 / 2}^{R}+\lambda_{y}\left(g_{u u}\right)_{i-1 / 2, j-1 / 2}^{C} \delta_{i-1 / 2, j-1 / 2}^{U, C}\right],
\end{aligned}
$$




$$
\begin{aligned}
& \frac{\partial H}{\partial u_{i-1 / 2, j-1 / 2}^{R}}=\frac{1}{4} \frac{\partial U_{L D}}{\partial u_{i-1 / 2, j-1 / 2}^{R}}+\frac{\lambda_{x}}{4} \frac{\partial F_{L D}}{\partial u_{i-1 / 2, j-1 / 2}^{R}}+\frac{\lambda_{y}}{4} \frac{\partial G_{D L}}{\partial u_{i-1 / 2, j-1 / 2}^{R}} \\
& =\frac{1}{16}\left[1+\lambda_{x}\left(f_{u}\right)_{i-1 / 2, j-1 / 2}^{C}+\lambda_{y}\left(g_{u}\right)_{i-1 / 2, j-1 / 2}^{C}\right] \\
& \times\left[1-\lambda_{x}\left(f_{u}\right)_{i-1 / 2, j-1 / 2}^{C}+\lambda_{y}\left(g_{u}\right)_{i-1 / 2, j-1 / 2}^{C}\right], \\
& \frac{\partial H}{\partial u_{i-1 / 2, j-1 / 2}^{U}}=\frac{1}{4} \frac{\partial U_{L D}}{\partial u_{i-1 / 2, j-1 / 2}^{U}}+\frac{\lambda_{x}}{4} \frac{\partial F_{L D}}{\partial u_{i-1 / 2, j-1 / 2}^{U}}+\frac{\lambda_{y}}{4} \frac{\partial G_{D L}}{\partial u_{i-1 / 2, j-1 / 2}^{U}} \\
& =\frac{1}{16}\left[1+\lambda_{x}\left(f_{u}\right)_{i-1 / 2, j-1 / 2}^{C}+\lambda_{y}\left(g_{u}\right)_{i-1 / 2, j-1 / 2}^{C}\right] \\
& \times\left[1+\lambda_{x}\left(f_{u}\right)_{i-1 / 2, j-1 / 2}^{C}-\lambda_{y}\left(g_{u}\right)_{i-1 / 2, j-1 / 2}^{C}\right], \\
& \frac{\partial H}{\partial u_{i-1 / 2, j-1 / 2}^{R U}}=\frac{1}{4} \frac{\partial U_{L D}}{\partial u_{i-1 / 2, j-1 / 2}^{R U}}+\frac{\lambda_{x}}{4} \frac{\partial F_{L D}}{\partial u_{i-1 / 2, j-1 / 2}^{R U}}+\frac{\lambda_{y}}{4} \frac{\partial G_{D L}}{\partial u_{i-1 / 2, j-1 / 2}^{R U}} \\
& =\frac{1}{16}\left[1+\lambda_{x}\left(f_{u}\right)_{i-1 / 2, j-1 / 2}^{C}+\lambda_{y}\left(g_{u}\right)_{i-1 / 2, j-1 / 2}^{C}\right] \\
& \times\left[1-\lambda_{x}\left(f_{u}\right)_{i-1 / 2, j-1 / 2}^{C}-\lambda_{y}\left(g_{u}\right)_{i-1 / 2, j-1 / 2}^{C}\right], \\
& \frac{\partial H}{\partial u_{i+1 / 2, j-1 / 2}^{C}}=\frac{1}{4} \frac{\partial U_{R D}}{\partial u_{i+1 / 2, j-1 / 2}^{C}}-\frac{\lambda_{x}}{4} \frac{\partial F_{R D}}{\partial u_{i+1 / 2, j-1 / 2}^{C}}+\frac{\lambda_{y}}{4} \frac{\partial G_{D R}}{\partial u_{i+1 / 2, j-1 / 2}^{C}} \\
& =\frac{1}{16}\left[1-\lambda_{x}\left(f_{u}\right)_{i+1 / 2, j-1 / 2}^{C}+\lambda_{y}\left(g_{u}\right)_{i+1 / 2, j-1 / 2}^{C}\right]^{2} \\
& -\frac{\lambda_{x}}{8}\left(f_{u u}\right)_{i+1 / 2, j-1 / 2}^{C} \delta_{i+1 / 2, j-1 / 2}^{U, C}-\frac{\lambda_{y}}{8}\left(g_{u u}\right)_{i+1 / 2, j-1 / 2}^{C} \delta_{i+1 / 2, j-1 / 2}^{C, L} \\
& +\frac{1}{8}\left[\lambda_{x}\left(f_{u u}\right)_{i+1 / 2, j-1 / 2}^{C}-\lambda_{y}\left(g_{u u}\right)_{i+1 / 2, j-1 / 2}^{C}\right] \\
& \times\left[\lambda_{x}\left(f_{u}\right)_{i+1 / 2, j-1 / 2}^{C} \delta_{i+1 / 2, j-1 / 2}^{C, L}+\lambda_{y}\left(g_{u}\right)_{i+1 / 2, j-1 / 2}^{C} \delta_{i+1 / 2, j-1 / 2}^{U, C}\right] \\
& +\frac{1}{8}\left[\lambda_{x}\left(f_{u}\right)_{i+1 / 2, j-1 / 2}^{C}-\lambda_{y}\left(g_{u}\right)_{i+1 / 2, j-1 / 2}^{C}\right] \\
& \times\left[\lambda_{x}\left(f_{u u}\right)_{i+1 / 2, j-1 / 2}^{C} \delta_{i+1 / 2, j-1 / 2}^{C, L}+\lambda_{y}\left(g_{u u}\right)_{i+1 / 2, j-1 / 2}^{C} \delta_{i+1 / 2, j-1 / 2}^{U, C}\right], \\
& \frac{\partial H}{\partial u_{i+1 / 2, j-1 / 2}^{L}}=\frac{1}{4} \frac{\partial U_{R D}}{\partial u_{i+1 / 2, j-1 / 2}^{L}}-\frac{\lambda_{x}}{4} \frac{\partial F_{R D}}{\partial u_{i+1 / 2, j-1 / 2}^{L}}+\frac{\lambda_{y}}{4} \frac{\partial G_{D R}}{\partial u_{i+1 / 2, j-1 / 2}^{L}} \\
& =\frac{1}{16}\left[1+\lambda_{x}\left(f_{u}\right)_{i+1 / 2, j-1 / 2}^{C}+\lambda_{y}\left(g_{u}\right)_{i+1 / 2, j-1 / 2}^{C}\right] \\
& \times\left[1-\lambda_{x}\left(f_{u}\right)_{i+1 / 2, j-1 / 2}^{C}+\lambda_{y}\left(g_{u}\right)_{i+1 / 2, j-1 / 2}^{C}\right],
\end{aligned}
$$




$$
\begin{aligned}
& \frac{\partial H}{\partial u_{i+1 / 2, j-1 / 2}^{U}}=\frac{1}{4} \frac{\partial U_{R D}}{\partial u_{i+1 / 2, j-1 / 2}^{U}}-\frac{\lambda_{x}}{4} \frac{\partial F_{R D}}{\partial u_{i+1 / 2, j-1 / 2}^{U}}+\frac{\lambda_{y}}{4} \frac{\partial G_{D R}}{\partial u_{i+1 / 2, j-1 / 2}^{U}} \\
& =\frac{1}{16}\left[1-\lambda_{x}\left(f_{u}\right)_{i+1 / 2, j-1 / 2}^{C}+\lambda_{y}\left(g_{u}\right)_{i+1 / 2, j-1 / 2}^{C}\right] \\
& \times\left[1-\lambda_{x}\left(f_{u}\right)_{i+1 / 2, j-1 / 2}^{C}-\lambda_{y}\left(g_{u}\right)_{i+1 / 2, j-1 / 2}^{C}\right], \\
& \frac{\partial H}{\partial u_{i+1 / 2, j-1 / 2}^{L U}}=\frac{1}{4} \frac{\partial U_{R D}}{\partial u_{i+1 / 2, j-1 / 2}^{L U}}-\frac{\lambda_{x}}{4} \frac{\partial F_{R D}}{\partial u_{i+1 / 2, j-1 / 2}^{L U}}+\frac{\lambda_{y}}{4} \frac{\partial G_{D R}}{\partial u_{i+1 / 2, j-1 / 2}^{L U}} \\
& =\frac{1}{16}\left[1+\lambda_{x}\left(f_{u}\right)_{i+1 / 2, j-1 / 2}^{C}-\lambda_{y}\left(g_{u}\right)_{i+1 / 2, j-1 / 2}^{C}\right] \\
& \times\left[1-\lambda_{x}\left(f_{u}\right)_{i+1 / 2, j-1 / 2}^{C}+\lambda_{y}\left(g_{u}\right)_{i+1 / 2, j-1 / 2}^{C}\right], \\
& \frac{\partial H}{\partial u_{i+1 / 2, j+1 / 2}^{C}}=\frac{1}{4} \frac{\partial U_{R U}}{\partial u_{i+1 / 2, j+1 / 2}^{C}}-\frac{\lambda_{x}}{4} \frac{\partial F_{R U}}{\partial u_{i+1 / 2, j+1 / 2}^{C}}-\frac{\lambda_{y}}{4} \frac{\partial G_{U R}}{\partial u_{i+1 / 2, j+1 / 2}^{C}} \\
& =\frac{1}{16}\left[1-\lambda_{x}\left(f_{u}\right)_{i+1 / 2, j+1 / 2}^{C}-\lambda_{y}\left(g_{u}\right)_{i+1 / 2, j+1 / 2}^{C}\right]^{2} \\
& +\frac{\lambda_{x}}{8}\left(f_{u u}\right)_{i+1 / 2, j+1 / 2}^{C} \delta_{i+1 / 2, j+1 / 2}^{C, D}+\frac{\lambda_{y}}{8}\left(g_{u u}\right)_{i+1 / 2, j+1 / 2}^{C} \delta_{i+1 / 2, j+1 / 2}^{C, L} \\
& +\frac{1}{8}\left[\lambda_{x}\left(f_{u u}\right)_{i+1 / 2, j+1 / 2}^{C}+\lambda_{y}\left(g_{u u}\right)_{i+1 / 2, j+1 / 2}^{C}\right] \\
& \times\left[\lambda_{x}\left(f_{u}\right)_{i+1 / 2, j+1 / 2}^{C} \delta_{i+1 / 2, j+1 / 2}^{C, L}+\lambda_{y}\left(g_{u}\right)_{i+1 / 2, j+1 / 2}^{C} \delta_{i+1 / 2, j+1 / 2}^{C, D}\right] \\
& +\frac{1}{8}\left[\lambda_{x}\left(f_{u}\right)_{i+1 / 2, j+1 / 2}^{C}+\lambda_{y}\left(g_{u}\right)_{i+1 / 2, j+1 / 2}^{C}\right] \\
& \times\left[\lambda_{x}\left(f_{u u}\right)_{i+1 / 2, j+1 / 2}^{C} \delta_{i+1 / 2, j+1 / 2}^{C, L}+\lambda_{y}\left(g_{\text {uu }}\right)_{i+1 / 2, j+1 / 2}^{C} \delta_{i+1 / 2, j+1 / 2}^{C, D}\right], \\
& \frac{\partial H}{\partial u_{i+1 / 2, j+1 / 2}^{L}}=\frac{1}{4} \frac{\partial U_{R U}}{\partial u_{i+1 / 2, j+1 / 2}^{L}}-\frac{\lambda_{x}}{4} \frac{\partial F_{R U}}{\partial u_{i+1 / 2, j+1 / 2}^{L}}-\frac{\lambda_{y}}{4} \frac{\partial G_{U R}}{\partial u_{i+1 / 2, j+1 / 2}^{L}} \\
& =\frac{1}{16}\left[1+\lambda_{x}\left(f_{u}\right)_{i+1 / 2, j+1 / 2}^{C}-\lambda_{y}\left(g_{u}\right)_{i+1 / 2, j+1 / 2}^{C}\right] \\
& \times\left[1-\lambda_{x}\left(f_{u}\right)_{i+1 / 2, j+1 / 2}^{C}-\lambda_{y}\left(g_{u}\right)_{i+1 / 2, j+1 / 2}^{C}\right], \\
& \frac{\partial H}{\partial u_{i+1 / 2, j+1 / 2}^{D}}=\frac{1}{4} \frac{\partial U_{R U}}{\partial u_{i+1 / 2, j+1 / 2}^{D}}-\frac{\lambda_{x}}{4} \frac{\partial F_{R U}}{\partial u_{i+1 / 2, j+1 / 2}^{D}}-\frac{\lambda_{y}}{4} \frac{\partial G_{U R}}{\partial u_{i+1 / 2, j+1 / 2}^{D}} \\
& =\frac{1}{16}\left[1-\lambda_{x}\left(f_{u}\right)_{i+1 / 2, j+1 / 2}^{C}+\lambda_{y}\left(g_{u}\right)_{i+1 / 2, j+1 / 2}^{C}\right] \\
& \times\left[1-\lambda_{x}\left(f_{u}\right)_{i+1 / 2, j+1 / 2}^{C}-\lambda_{y}\left(g_{u}\right)_{i+1 / 2, j+1 / 2}^{C}\right],
\end{aligned}
$$




$$
\begin{aligned}
& \frac{\partial H}{\partial u_{i+1 / 2, j+1 / 2}^{L D}}=\frac{1}{4} \frac{\partial U_{R U}}{\partial u_{i+1 / 2, j+1 / 2}^{L D}}-\frac{\lambda_{x}}{4} \frac{\partial F_{R U}}{\partial u_{i+1 / 2, j+1 / 2}^{L D}}-\frac{\lambda_{y}}{4} \frac{\partial G_{U R}}{\partial u_{i+1 / 2, j+1 / 2}^{L D}} \\
& =\frac{1}{16}\left[1+\lambda_{x}\left(f_{u}\right)_{i+1 / 2, j+1 / 2}^{C}+\lambda_{y}\left(g_{u}\right)_{i+1 / 2, j+1 / 2}^{C}\right] \\
& \times\left[1-\lambda_{x}\left(f_{u}\right)_{i+1 / 2, j+1 / 2}^{C}-\lambda_{y}\left(g_{u}\right)_{i+1 / 2, j+1 / 2}^{C}\right], \\
& \frac{\partial H}{\partial u_{i-1 / 2, j+1 / 2}^{C}}=\frac{1}{4} \frac{\partial U_{L U}}{\partial u_{i-1 / 2, j+1 / 2}^{C}}-\frac{\lambda_{x}}{4} \frac{\partial F_{L U}}{\partial u_{i-1 / 2, j+1 / 2}^{C}}-\frac{\lambda_{y}}{4} \frac{\partial G_{U L}}{\partial u_{i-1 / 2, j+1 / 2}^{C}} \\
& =\frac{1}{16}\left[1+\lambda_{x}\left(f_{u}\right)_{i-1 / 2, j+1 / 2}^{C}-\lambda_{y}\left(g_{u}\right)_{i-1 / 2, j+1 / 2}^{C}\right]^{2} \\
& -\frac{\lambda_{x}}{8}\left(f_{\text {uu }}\right)_{i-1 / 2, j+1 / 2}^{C} \delta_{i-1 / 2, j+1 / 2}^{C, D}-\frac{\lambda_{y}}{8}\left(g_{u u}\right)_{i-1 / 2, j+1 / 2}^{C} \delta_{i-1 / 2, j+1 / 2}^{R, C} \\
& -\frac{1}{8}\left[\lambda_{x}\left(f_{u u}\right)_{i-1 / 2, j+1 / 2}^{C}-\lambda_{y}\left(g_{u u}\right)_{i-1 / 2, j+1 / 2}^{C}\right] \\
& \times\left[\lambda_{x}\left(f_{u}\right)_{i-1 / 2, j+1 / 2}^{C} \delta_{i-1 / 2, j+1 / 2}^{R, C}+\lambda_{y}\left(g_{u}\right)_{i-1 / 2, j+1 / 2}^{C} \delta_{i-1 / 2, j+1 / 2}^{C, D}\right] \\
& -\frac{1}{8}\left[\lambda_{x}\left(f_{u}\right)_{i-1 / 2, j+1 / 2}^{C}-\lambda_{y}\left(g_{u}\right)_{i-1 / 2, j+1 / 2}^{C}\right] \\
& \times\left[\lambda_{x}\left(f_{u u}\right)_{i-1 / 2, j+1 / 2}^{C} \delta_{i-1 / 2, j+1 / 2}^{R, C}+\lambda_{y}\left(g_{u u}\right)_{i-1 / 2, j+1 / 2}^{C} \delta_{i-1 / 2, j+1 / 2}^{C, D}\right], \\
& \frac{\partial H}{\partial u_{i-1 / 2, j+1 / 2}^{R}}=\frac{1}{4} \frac{\partial U_{L U}}{\partial u_{i-1 / 2, j+1 / 2}^{R}}-\frac{\lambda_{x}}{4} \frac{\partial F_{L U}}{\partial u_{i-1 / 2, j+1 / 2}^{R}}-\frac{\lambda_{y}}{4} \frac{\partial G_{U L}}{\partial u_{i-1 / 2, j+1 / 2}^{R}} \\
& =\frac{1}{16}\left[1+\lambda_{x}\left(f_{u}\right)_{i-1 / 2, j+1 / 2}^{C}-\lambda_{y}\left(g_{u}\right)_{i-1 / 2, j+1 / 2}^{C}\right] \\
& \times\left[1-\lambda_{x}\left(f_{u}\right)_{i-1 / 2, j+1 / 2}^{C}-\lambda_{y}\left(g_{u}\right)_{i-1 / 2, j+1 / 2}^{C}\right], \\
& \frac{\partial H}{\partial u_{i-1 / 2, j+1 / 2}^{D}}=\frac{1}{4} \frac{\partial U_{L U}}{\partial u_{i-1 / 2, j+1 / 2}^{D}}-\frac{\lambda_{x}}{4} \frac{\partial F_{L U}}{\partial u_{i-1 / 2, j+1 / 2}^{D}}-\frac{\lambda_{y}}{4} \frac{\partial G_{U L}}{\partial u_{i-1 / 2, j+1 / 2}^{D}} \\
& =\frac{1}{16}\left[1+\lambda_{x}\left(f_{u}\right)_{i-1 / 2, j+1 / 2}^{C}+\lambda_{y}\left(g_{u}\right)_{i-1 / 2, j+1 / 2}^{C}\right] \\
& \times\left[1+\lambda_{x}\left(f_{u}\right)_{i-1 / 2, j+1 / 2}^{C}-\lambda_{y}\left(g_{u}\right)_{i-1 / 2, j+1 / 2}^{C}\right], \\
& \frac{\partial H}{\partial u_{i-1 / 2, j+1 / 2}^{R D}}=\frac{1}{4} \frac{\partial U_{L U}}{\partial u_{i-1 / 2, j+1 / 2}^{R D}}-\frac{\lambda_{x}}{4} \frac{\partial F_{L U}}{\partial u_{i-1 / 2, j+1 / 2}^{R D}}-\frac{\lambda_{y}}{4} \frac{\partial G_{U L}}{\partial u_{i-1 / 2, j+1 / 2}^{R D}} \\
& =\frac{1}{16}\left[1+\lambda_{x}\left(f_{u}\right)_{i-1 / 2, j+1 / 2}^{C}-\lambda_{y}\left(g_{u}\right)_{i-1 / 2, j+1 / 2}^{C}\right] \\
& \times\left[1-\lambda_{x}\left(f_{u}\right)_{i-1 / 2, j+1 / 2}^{C}+\lambda_{y}\left(g_{u}\right)_{i-1 / 2, j+1 / 2}^{C}\right] .
\end{aligned}
$$

\section{References}


[1] X. Zhang, C. W. Shu, On maximum-principle-satisfying high order schemes for scalar conservation laws, J. Comput. Phys. 229 (2010) 3091-3120.

[2] B. Larrouturou, How to preserve the mass fractions positivity when computing compressible multi-component flows, J. Comput. Phys. 95 (1991) 59-84.

[3] S. Karni, Multicomponent flow calculations by a consistent primitive algorithm, J. Comput. Phys. 112 (1994) 31-43.

[4] R. Abgrall, How to prevent pressure oscillations in multicomponent flow calculations: a quasi conservative approach, J. Comput. Phys. 125 (1996) $150-160$.

[5] R. Abgrall, S. Karni, Computations of compressible multifluids, J. Comput. Phys. 169 (2001) 594-623.

[6] K. M. Shyue, An efficient shock-capturing algorithm for compressible multicomponent problems, J. Comput. Phys. 142 (1998) 208-242.

[7] K. M. Shyue, A fluid-mixture type algorithm for compressible multicomponent flow with van der Waals equation of state, J. Comput. Phys. 156 (1999) 43-88.

[8] K. M. Shyue, A fluid-mixture type algorithm for compressible multicomponent flow with Mie-Grüneisen equation of state, J. Comput. Phys. 171 (2001) 678-707.

[9] A. Harten, High resolution schemes for hyperbolic conservation laws, J. Comput. Phys. 49 (1983) 357-393.

[10]S. Osher, S. Chakravarthy, High resolution schemes and the entropy condition, SIAM J. Numer. Anal. 21 (1984) 955-984.

[11]S. C. Chang, The method of space-time conservation element and solution element-a new approach for solving the Navier-Stokes and Euler equations, J. Comput. Phys. 119 (1995) 295-324.

[12]S. C. Chang, X. Y. Wang and C. Y. Chow, The space-time conservation element and solution element method: a new high-resolution and genuinely multidimensional paradigm for solving conservation laws, J. Comput. Phys. 156 (1999) 89 136.

[13]Z. C. Zhang, S. T. John Yu, S. C. Chang, A space-time conservation element and solution element method for solving the two- and three-dimensional unsteady 
Euler equations using quadrilateral and hexahedral meshes, J. Comput. Phys. 175 (2002) 168-199.

[14]D. L. Bilyeu, S. T. J. Yu, Y. Y. Chen, J. L. Cambier, A two-dimensional fourth-order unstructured-meshed Euler solver based on the CESE method, J. Comput. Phys. 257 (2014) 981-999.

[15]H. Shen, C. Y. Wen, K. X. Liu, D. L. Zhang, Robust high-order space-time conservative schemes for solving conservation laws on hybrid meshes, J. Comput. Phys. 281 (2015) 375-402.

[16]H. Shen, C. Y. Wen, D. L. Zhang, A characteristic space-time conservation element and solution element method for conservation laws, J. Comput. Phys. 288 (2015) 101-118.

[17]H. Shen, C. Y. Wen, A characteristic space-time conservation element and solution element method for conservation laws II. multidimensional extension, J. Comput. Phys. 305 (2016) 775-792.

[18]S. Qamar, M. Ahmed, I. Ali, The space-time CE/SE method for solving reduced two-fluid flow model, Commun. Comput. Phys. 12 (2012) 1070-1095.

[19]C. W. Shu, S. Osher, Efficient implementation of essentially non-oscillatory shock-capturing schemes, J. Comput. Phys. 77 (1988) 439-471.

[20]A. Harten, B. Engquist, S. Osher, S. Chakravarthy, Uniformly high order essentially non-oscillatory schemes, III. J. Comput. Phys. 71 (1987) 231-303.

[21]V. A. Titarev, E. F. Toro, ADER: Arbitrary high order Godunov approach, J. Sci. Comput. 17 (2002) 609-618.

[22]S. C. Chang, Courant number insensitive CE/SE schemes, AIAA (2002) 2002-3890.

[23]W. Li, Y. X. Ren, G. Lei, H. Luo, The multi-dimensional limiters for solving hyperbolic conservation laws on unstructured grids, J. Comput. Phys. 230 (2011) 7775-7795.

[24]J. T. Wang, K. X. Liu, D. L. Zhang, A new CE/SE scheme for multi-material elastic-plastic flow and its application, Comput. Fluids 38 (2009) 544-551. 
[25]D. Ranjan, J. Oakley, R. Bonazza, shock-bubble interactions, Annu. Rev. Fluid Mech. 43 (2011) 117-140.

[26]T. G. Theofanous, Aerobreakup of Newtonian and Viscoelastic Liquids, Annu. Rev. Fluid Mech. 43 (2011) 661-690.

[27]C. W. Hirt, B. D. Nichols, Volume of fluid (VOF) method for the dynamics of free boundaries, J. Comput. Phys. 39 (1981) 201-225.

[28]R. Fedkiw, T. Aslam, B. Merriman, S. Osher, A non-oscillatory Eulerian approach to interfaces in multimaterial flows (the ghost fluid method), J. Comput. Phys. 152 (1999) 457-492.

[29]X. Y. Hu, B. C. Khoo, An interface interaction method for compressible multifluids, J. Comput. Phys. 198 (2004) 35-64.

[30]X. Y. Hu, B. C. Khoo, N. A. Adams, F. L. Huang, A conservative interface method for compressible flows, J. Comput. Phys. 219 (2006) 553-578.

[31]G. Tryggvason, G. Bunner, A. Esmaeeli, D. Juric, N. Al-Rawahi, W. Tauber, J. Han, S. Nas, Y. J. Jan, A front-tracking method for the computations of multiphase flow, J. Comput. Phys. 169 (2001) 708-759.

[32]H. Terashima, G. Tryggvason, A front-tracking/ghost-fluid method for fluid interfaces in compressible flows, J. Comput. Phys. 228 (2009) 4012-4037.

[33]M. R. Baer, J. W. Nunziato, A two-phase mixture theory for the deflagration-to-detonation transition (DDT) in reactive granular materials, J. Multiphase Flow 12 (1986) 861-889.

[34]R. Saurel, R. Abgrall, A simple method for compressible multifluid flows, SIAM J. Sci. Comput. 21 (1999) 1115-1145.

[35]G. Allaire, S. Clerc, S. Kokh, A five-equation model for the simulation of interfaces between compressible fluids, J. Comput. Phys. 181 (2002) 577-616.

[36]A. Murrone, H. Guillard, A five equation reduced model for compressible two phase flow problems, J. Comput. Phys. 202 (2004) 664-698.

[37]E. Johnsen, F. Ham, Preventing numerical errors generated by interface-capturing schemes in compressible multi-material flows, J. Comput. Phys. 231 (2012) $5705-5717$. 
[38]E. Johnsen, T. Colonius, Implementation of WENO schemes in compressible multicomponent flow problems, J. Comput. Phys. 219 (2006) 715-732.

[39]X. Y. Hu, N. A. Adams, G. Iaccarino, On the HLLC Riemann solver for interface interaction in compressible multi-fluid flow, J. Comput. Phys. 228 (2009) $6572-6589$.

[40]H. Luo, J. D. Baum, R. Löhner, On the computation of multi-material flows using ALE formulation, J. Comput. Phys. 194 (2004) 304-328.

[41]H. Chen, Two-dimensional simulation of stripping breakup of a water droplet, AIAA J. 46 (2008) 1135-1143.

[42]C. Laney, Computational gas dynamics, 1998.

[43]F. Ismail, P. L. Roe, Affordable, entropy-consistent Euler flux functions II: Entropy production at shocks, J. Comput. Phys. 228 (2009) 5410-5436.

[44]J. Massoni, R. Saurel, B. Nkonga, R. Abgrall, Propositions de méthodes et modèles Eulériens pour les problèmes à interfaces entre fluides compressibles en présence de transfert de chaleur, Int. J. Heat Mass Trans. 45 (2001) 1287-1307.

[45]C. H. Chang, M. S. Liou, A robust and accurate approach to computing compressible multiphase flow: Stratified flow model and AUSM+-up scheme, J. Comput. Phys. 225 (2007) 840-873.

[46]Y. Y. Niu, Y. C. Lin, C. H. Chang, A further work on multi-phase two-fluid approach for compressible multi-phase flows, Int. J. Numer. Meth. Fluids 58 (2008) 879-896.

[47]D. Igra, K. Takayama, A study of shock wave loading on a cylindrical water column, Reports of the Institute of Fluid Science, Tohoku University, 13 (2001) $19-36$. 


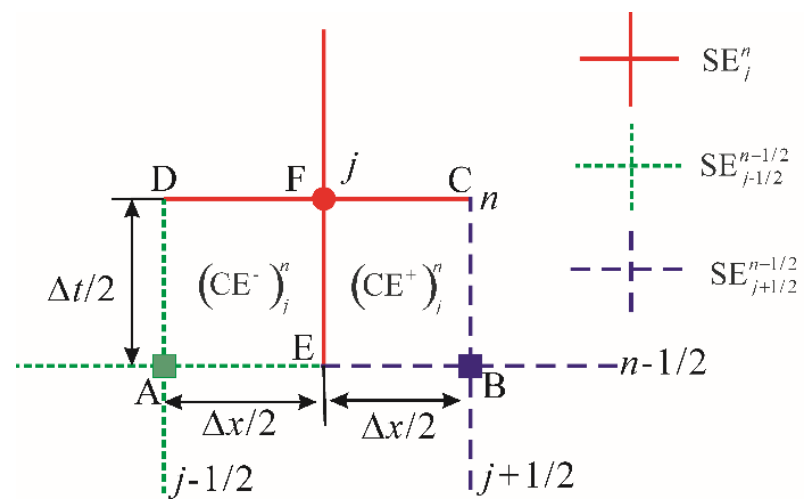

(a)

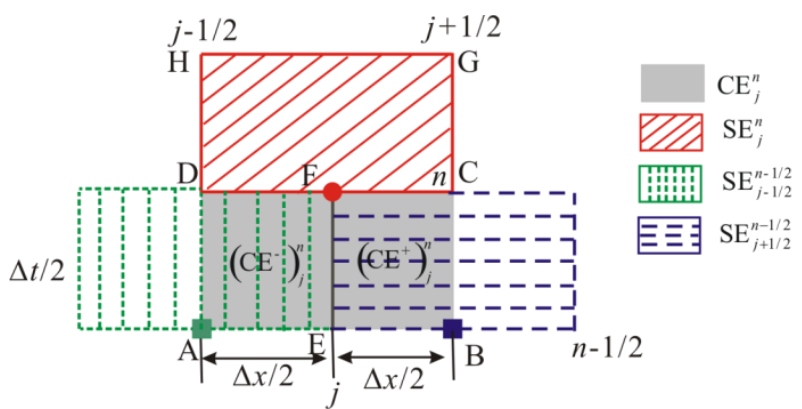

(b)

Figure 1. Schematics of CE and SE for (a) the central CE/SE scheme and (b) the upwind CE/SE scheme

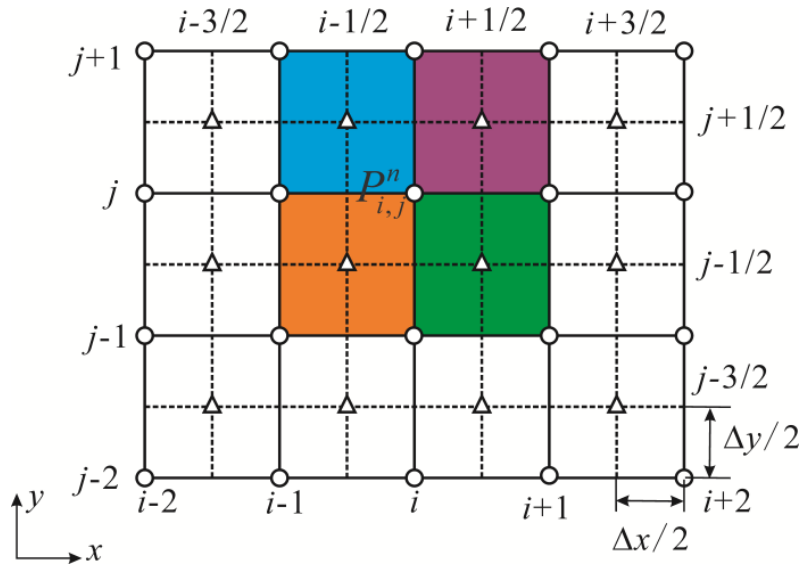

(a) Spatial mesh grids

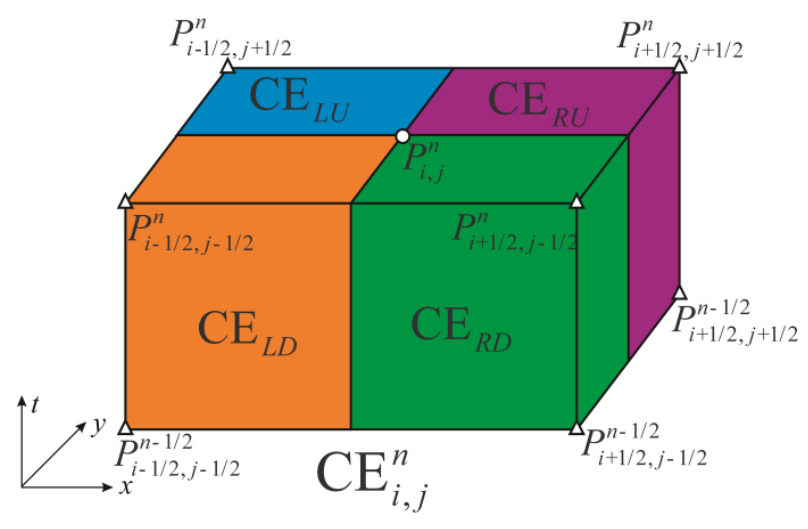

(c) Conservation element

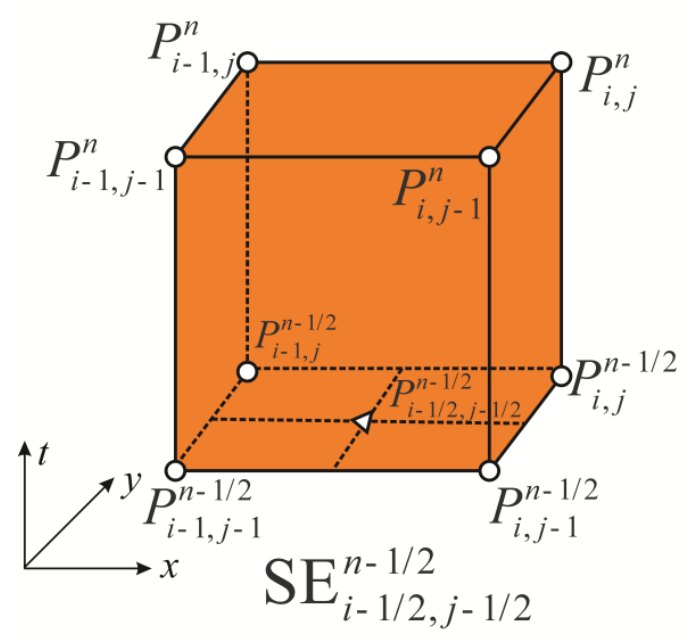

(b) Solution element

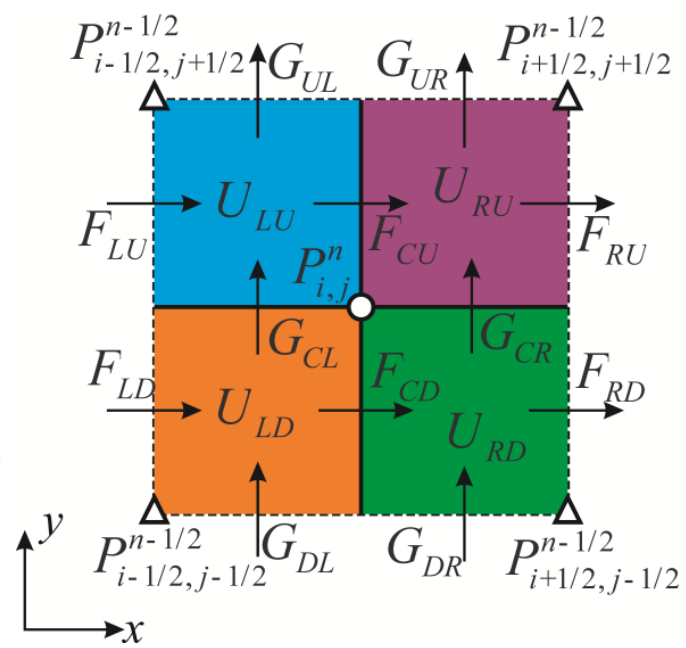

(d) Fluxes in one CE 
Figure 2. Schematics of (a) spatial mesh grids, (b) solution element, (c) conservation element and (d) corresponding fluxes in a $\mathrm{CE}$ for the $2 \mathrm{D}$ upwind $\mathrm{CE} / \mathrm{SE}$ scheme on rectangular meshes.

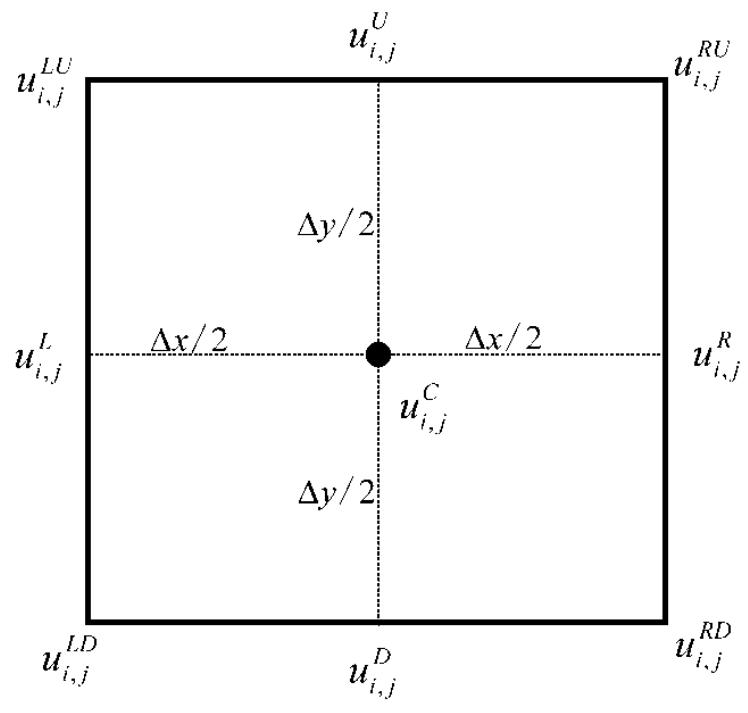

Figure 3. Traces of $u_{i, j}(x, y)$.

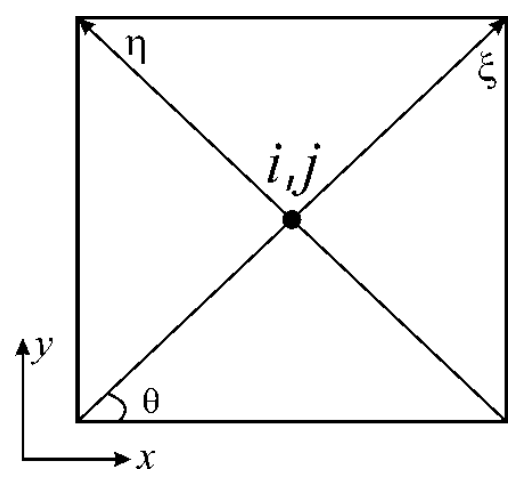

Figure 4. Definitions of $\xi-\eta$ coordinates.
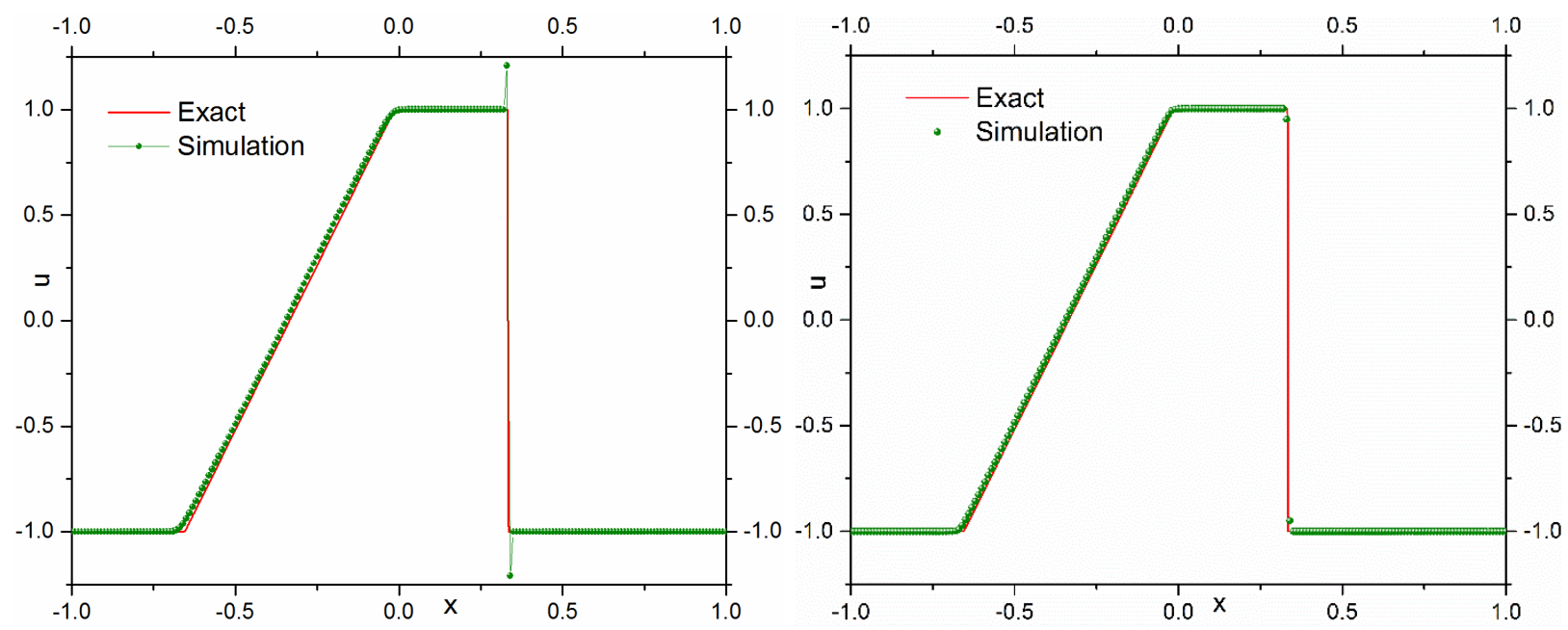

(a)

(b) 
Figure 5. Numerical solutions of rarefaction with a stationary shock at $t=0.32$ : (a) without limiter; (b) with limiter.

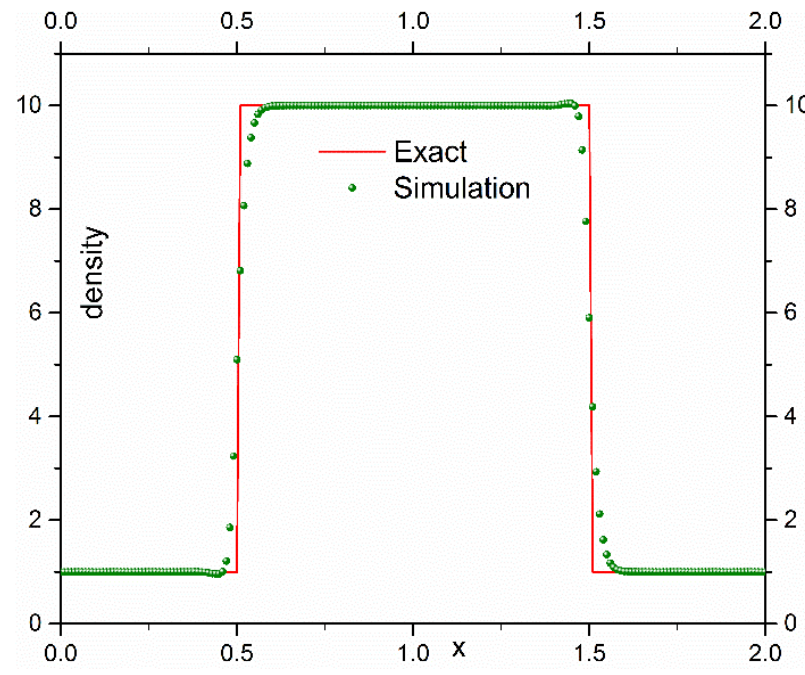

(a)

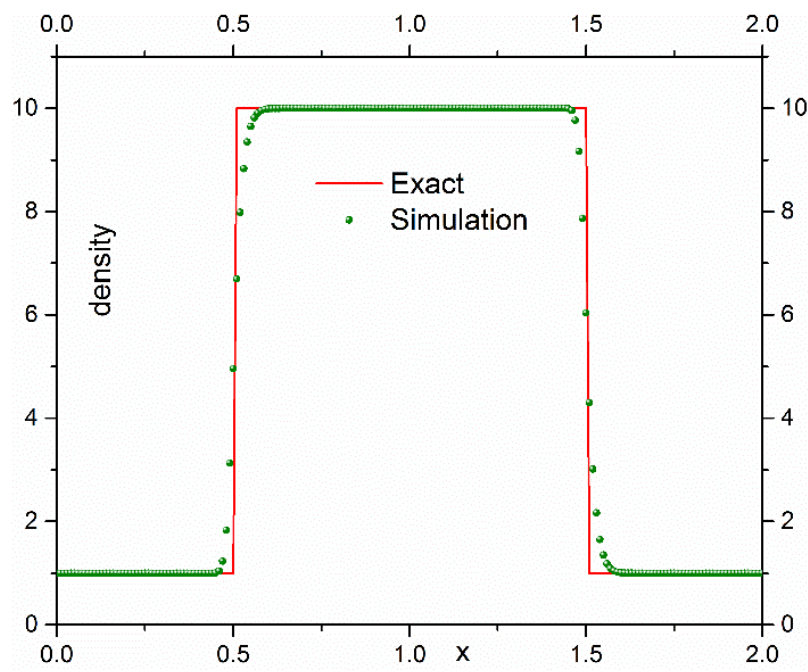

(b)

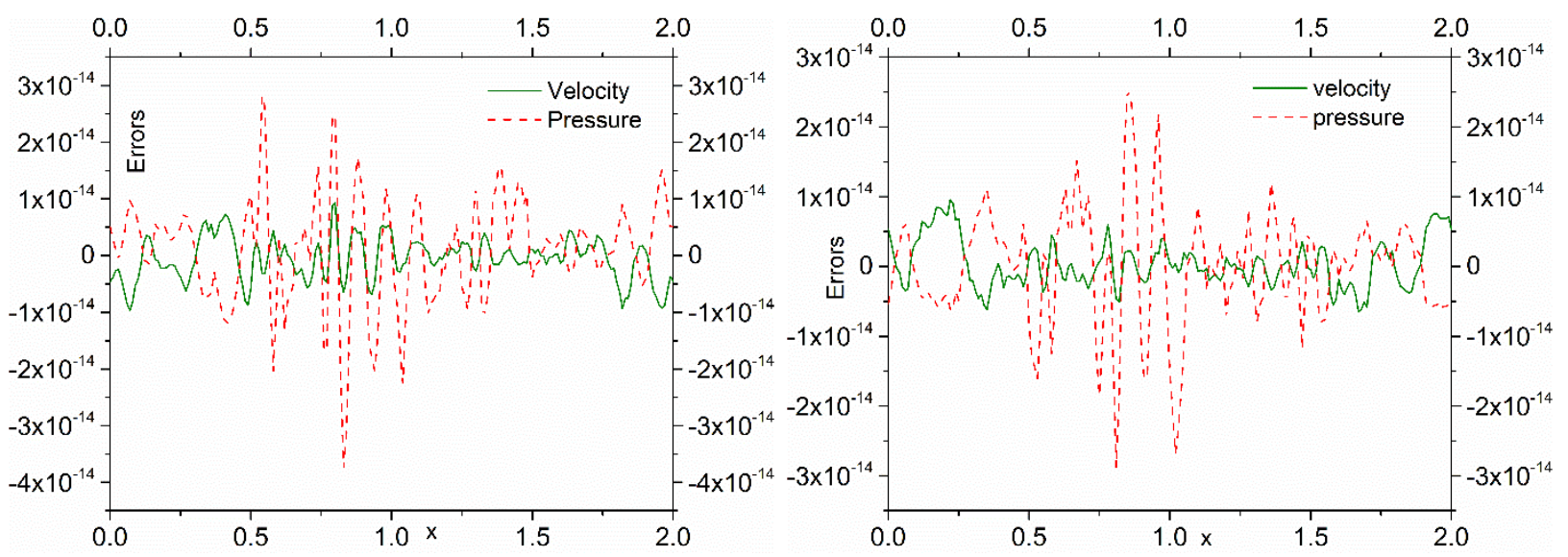

(c)

(d)

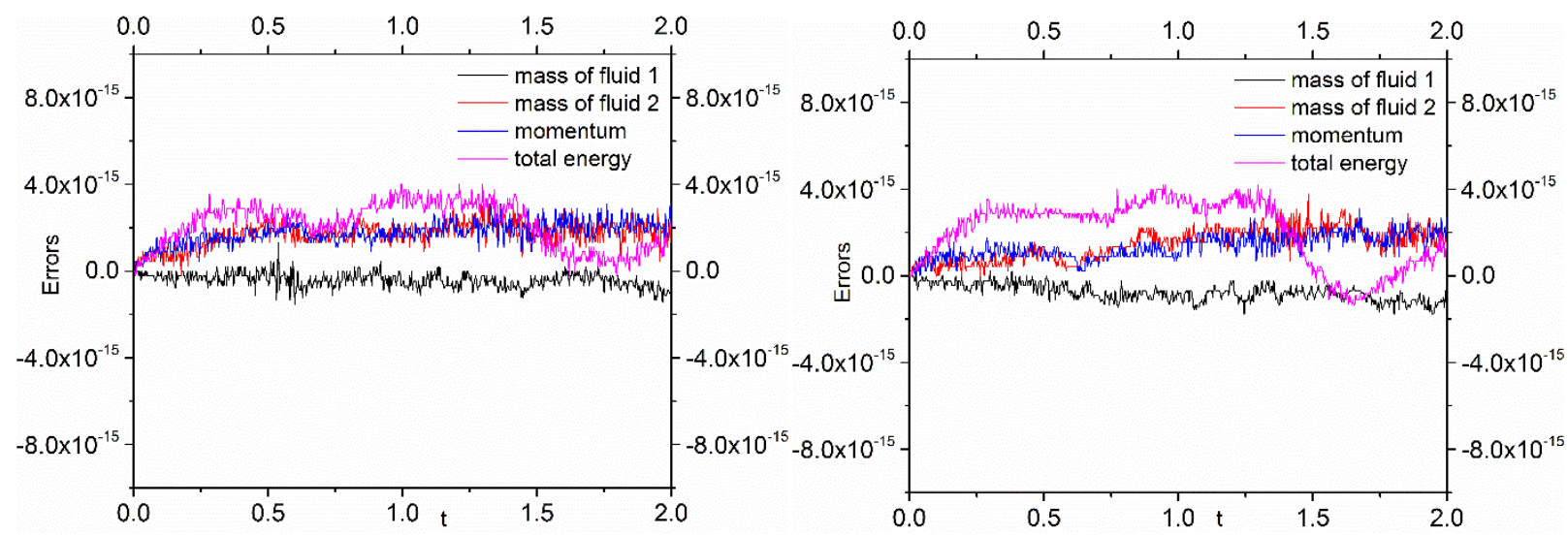

(e) 


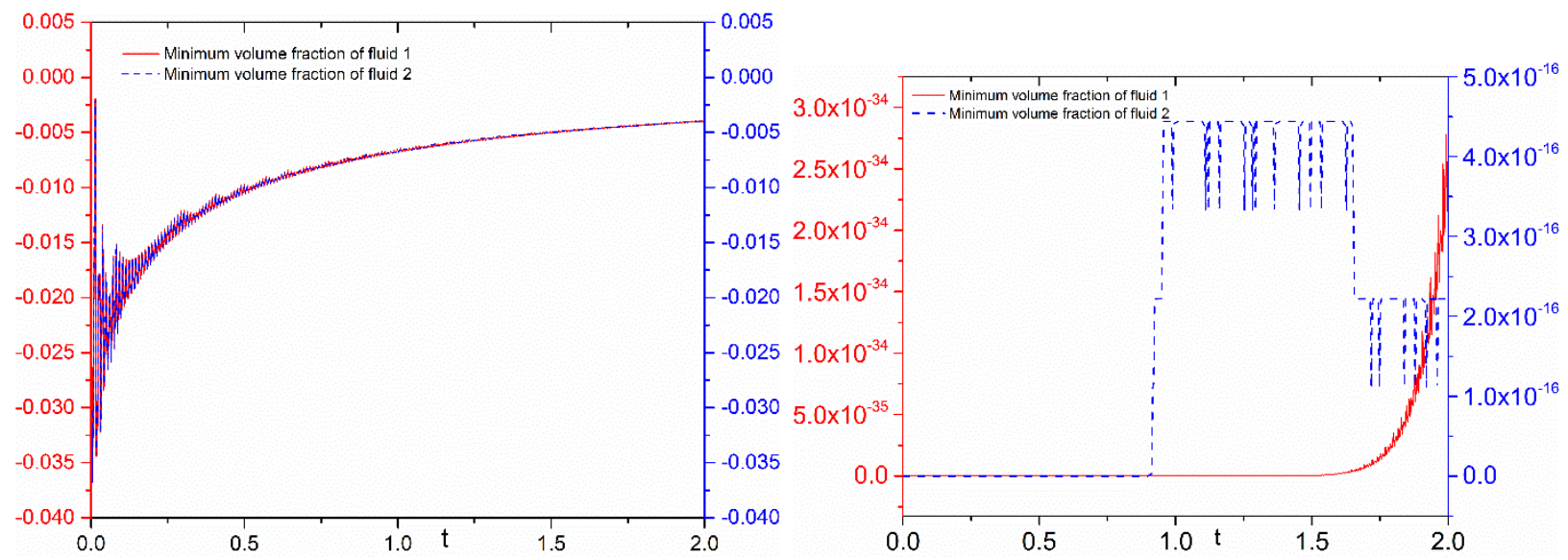

$(\mathrm{g})$

(h)

Figure 6 The density profile, errors of velocity and pressure, errors of conserved quantities and history of minimum volume fractions for the gas-gas interface propagation computed without (left column) and with (right column) the limiter.

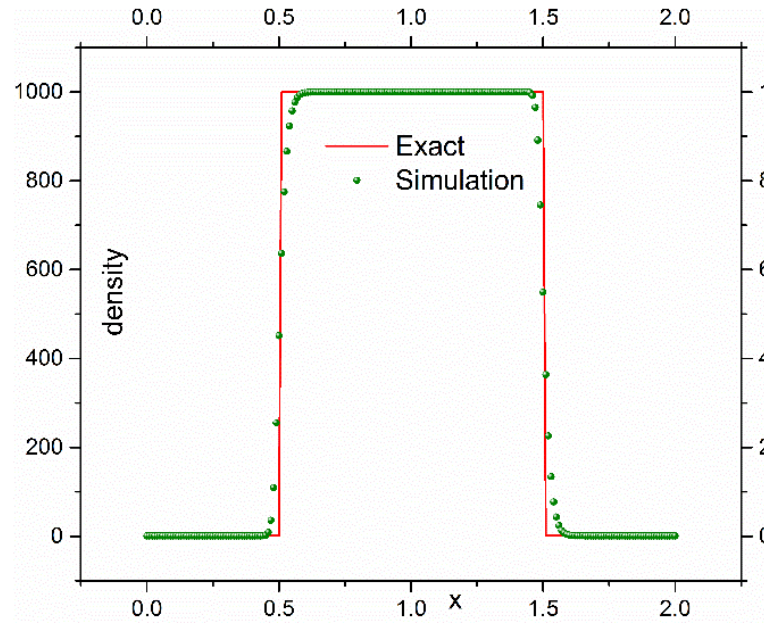

(a)

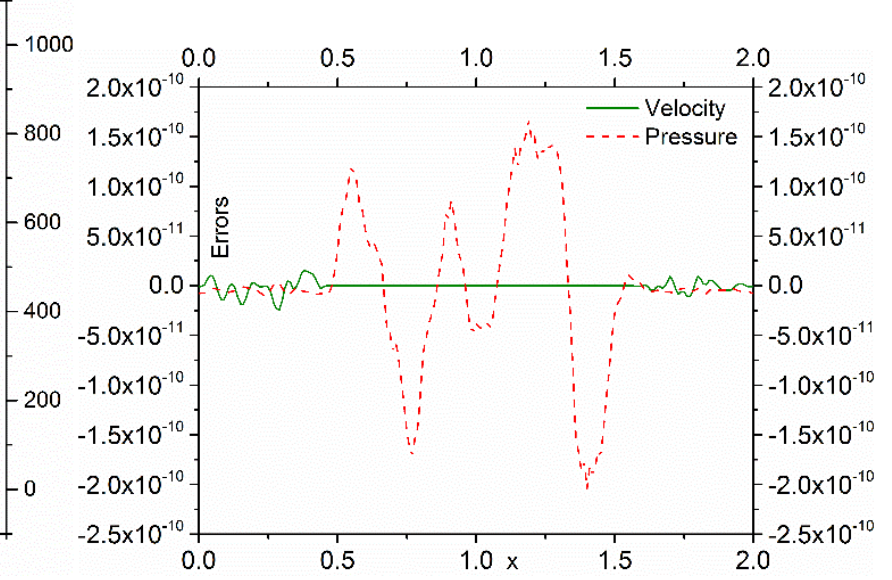

(b)

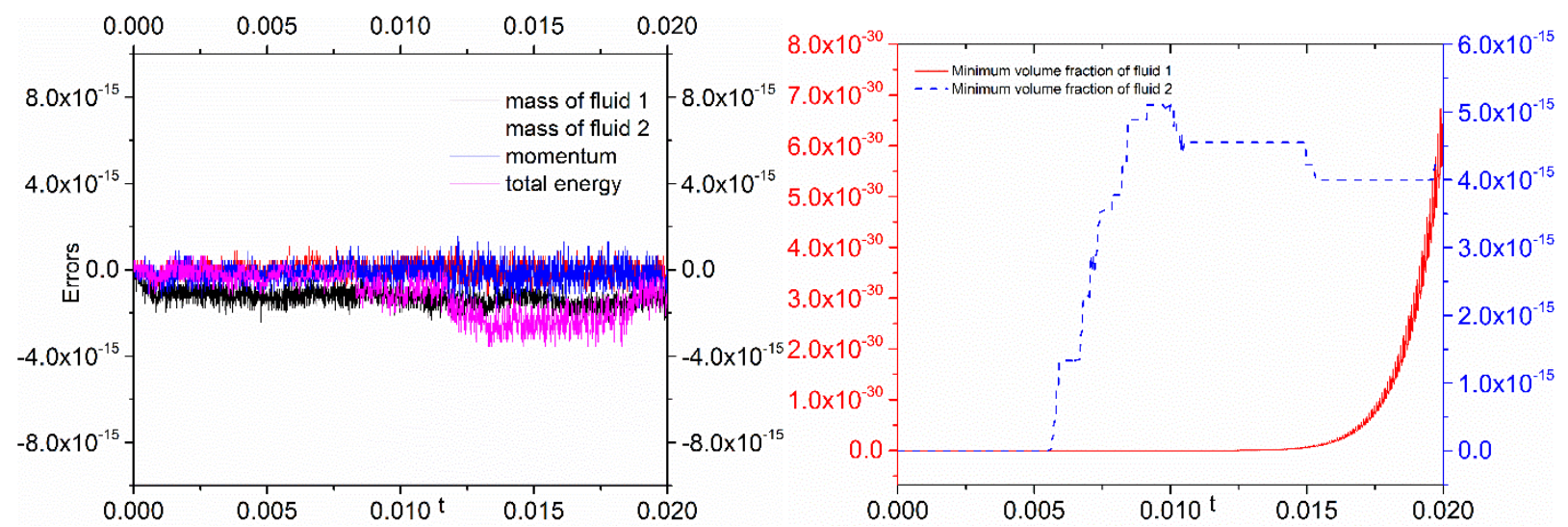


(c)

(d)

Figure 7. The (a) density profile, (b) errors of velocity and pressure, (c) errors of conserved quantities and (d) histories of minimum volume fractions for the propagation of the gas-water interface computed with the limiter.

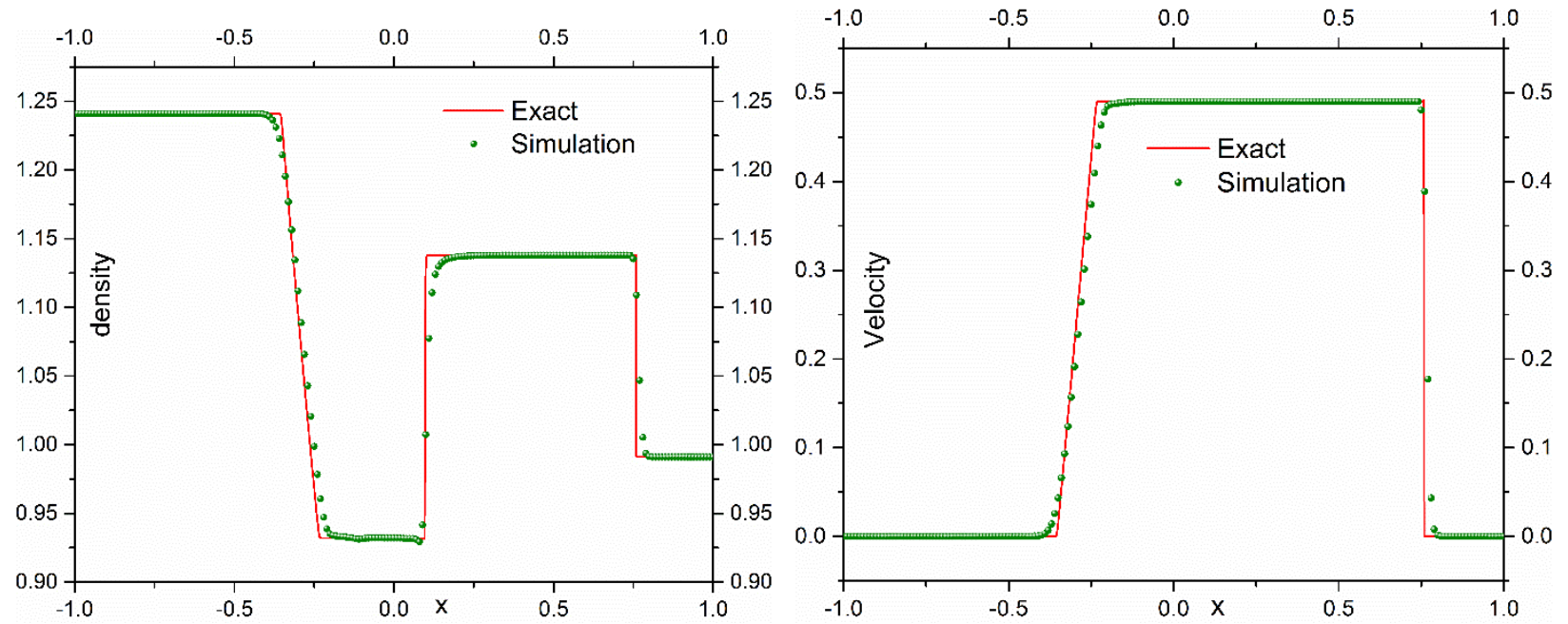

(a)

(b)

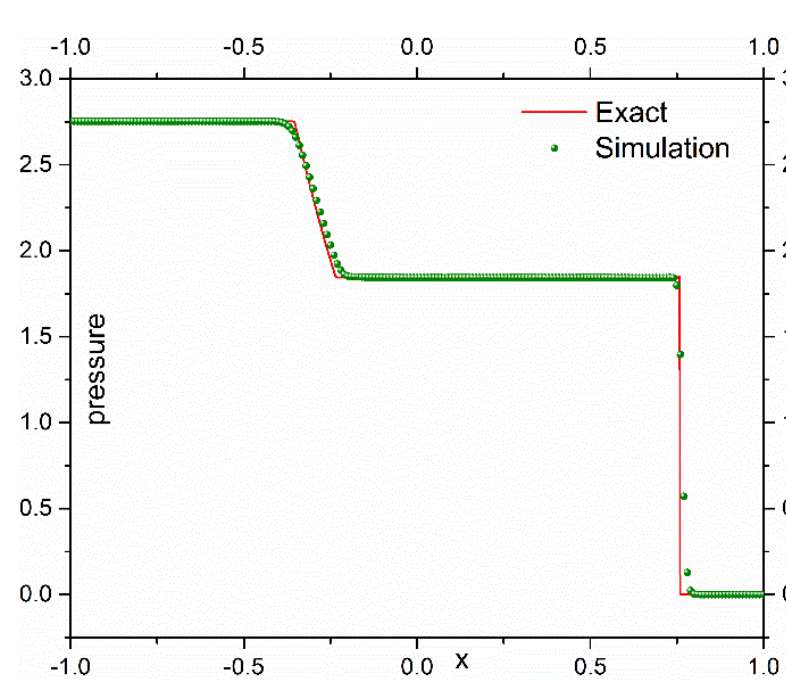

(c)

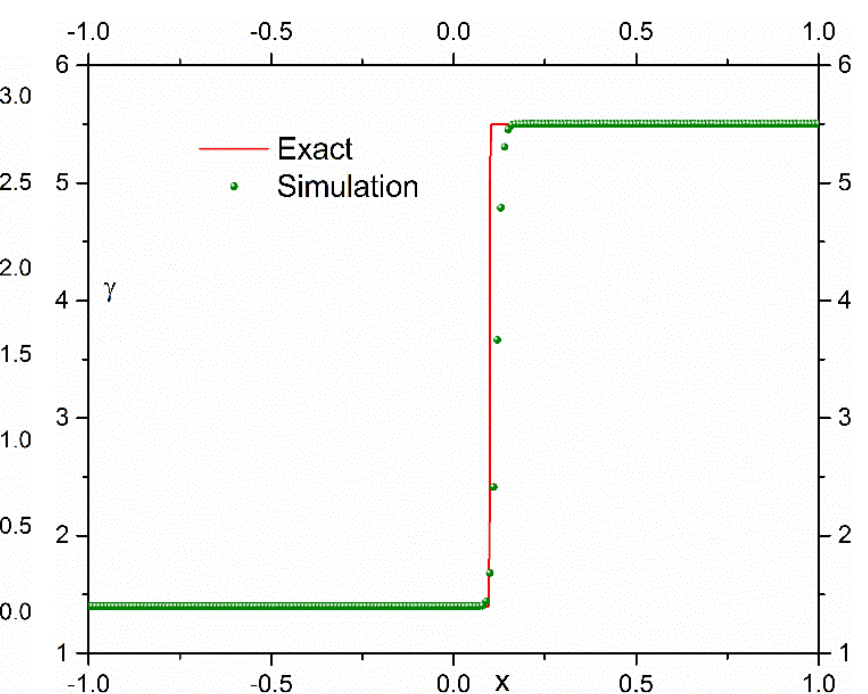

(d)

Figure 8. The (a) density, (b) velocity, (c) pressure and (d) $\gamma$ profiles of the gas-liquid Riemann problem, at $t=0.2$. 


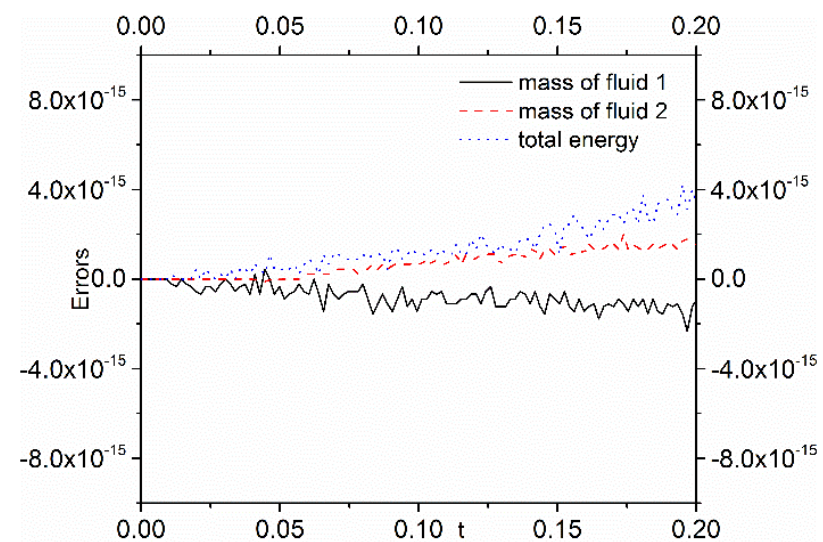

(a)

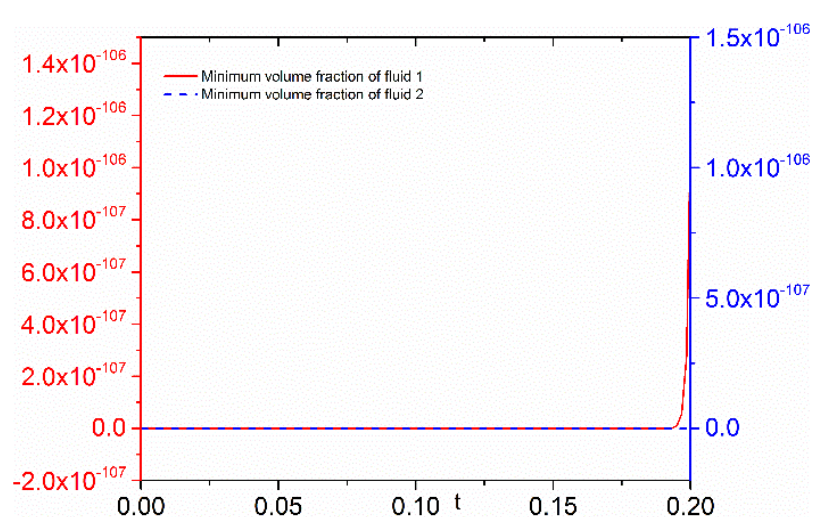

(b)

Figure 9. (a) The errors of conserved quantities and (b) the histories of minimum volume fractions for gas-liquid Riemann problem.

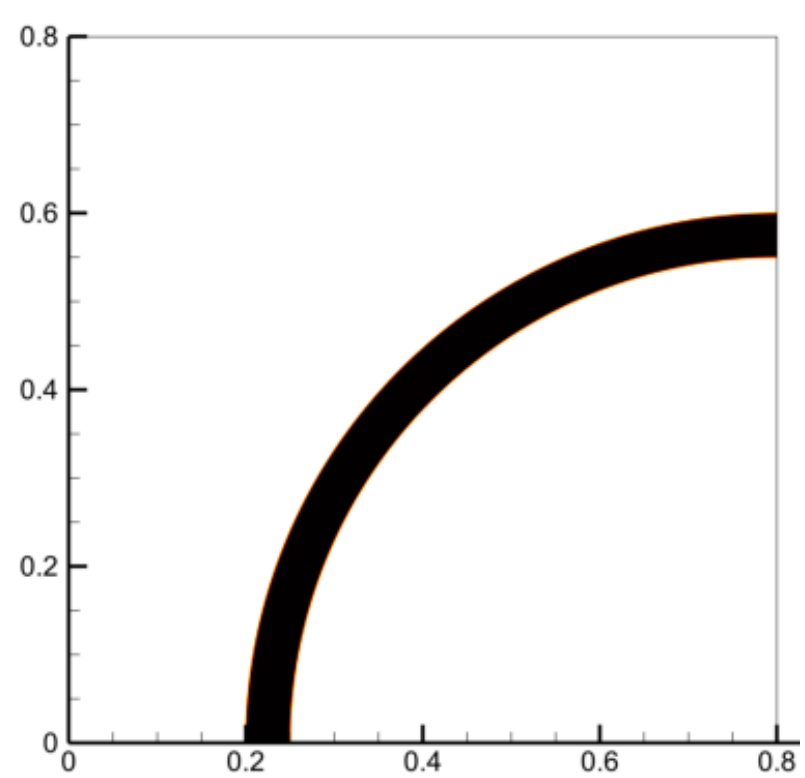

(a) $\mathrm{T}=0$

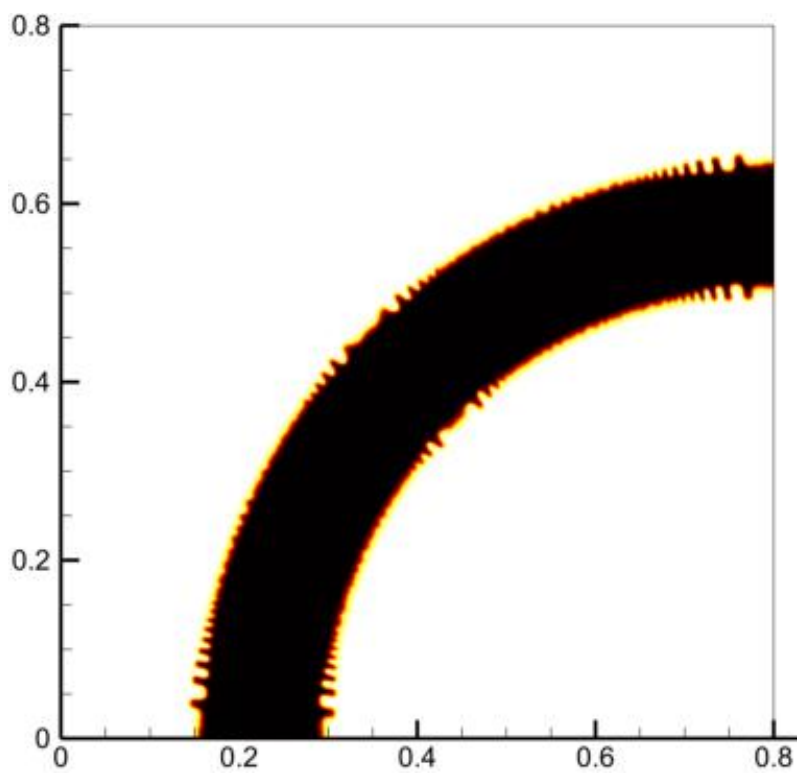

(b) $\mathrm{T}=0.64 \mathrm{~ms}$ 


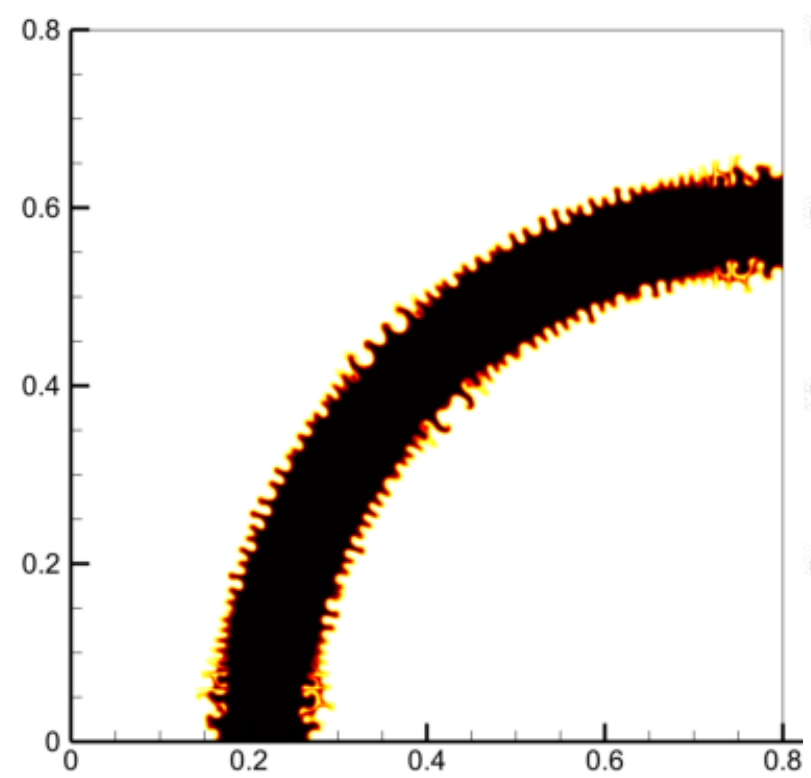

(c) $\mathrm{T}=1.545 \mathrm{~ms}$

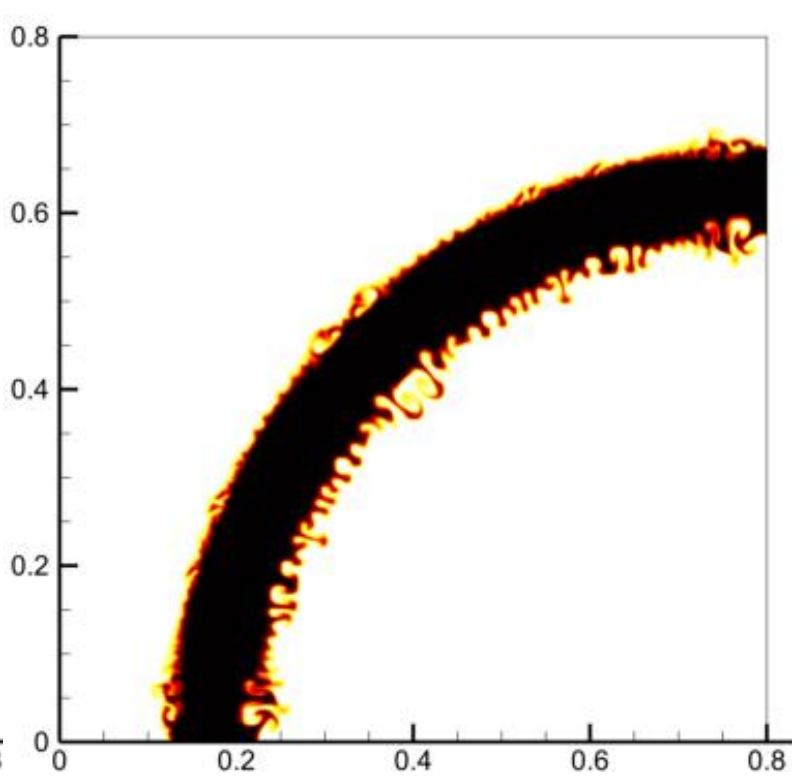

(d) $\mathrm{T}=2.523 \mathrm{~ms}$

Figure 10. The volume fraction evolution of heavy gas (black) for example 4.

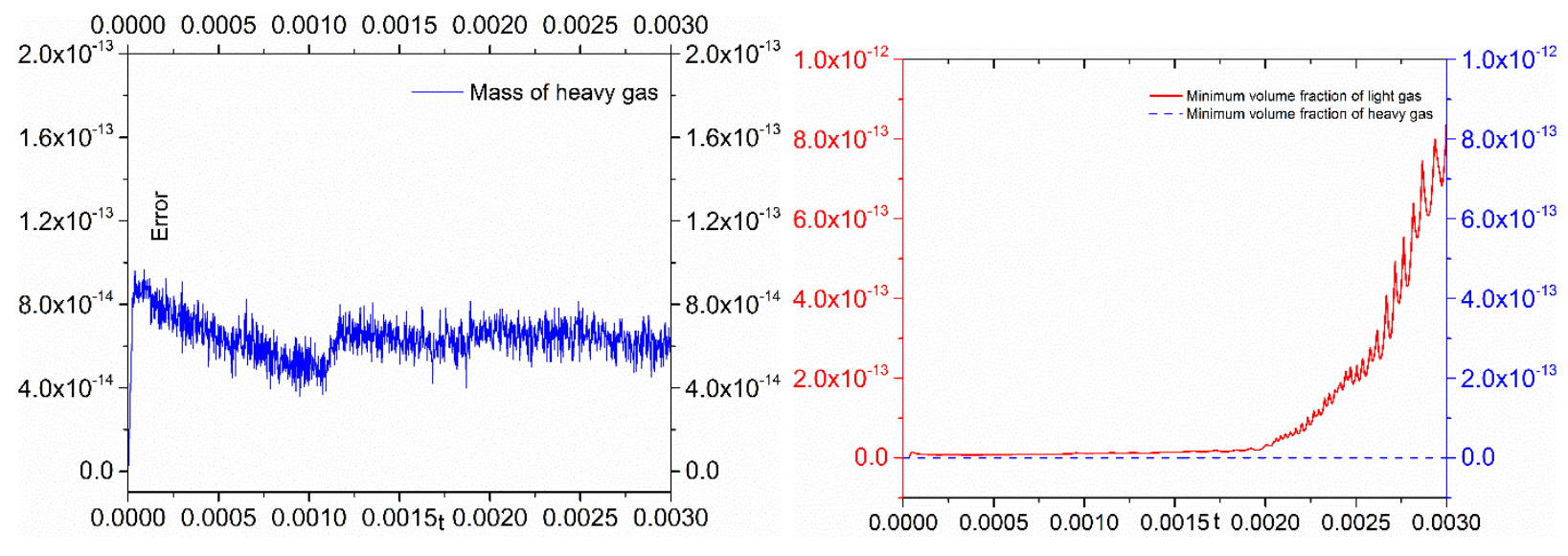

(a)

(b)

Figure 11. (a) The errors of the mass of the heavy gas and (b) the histories of minimum volume fractions for Example 4.

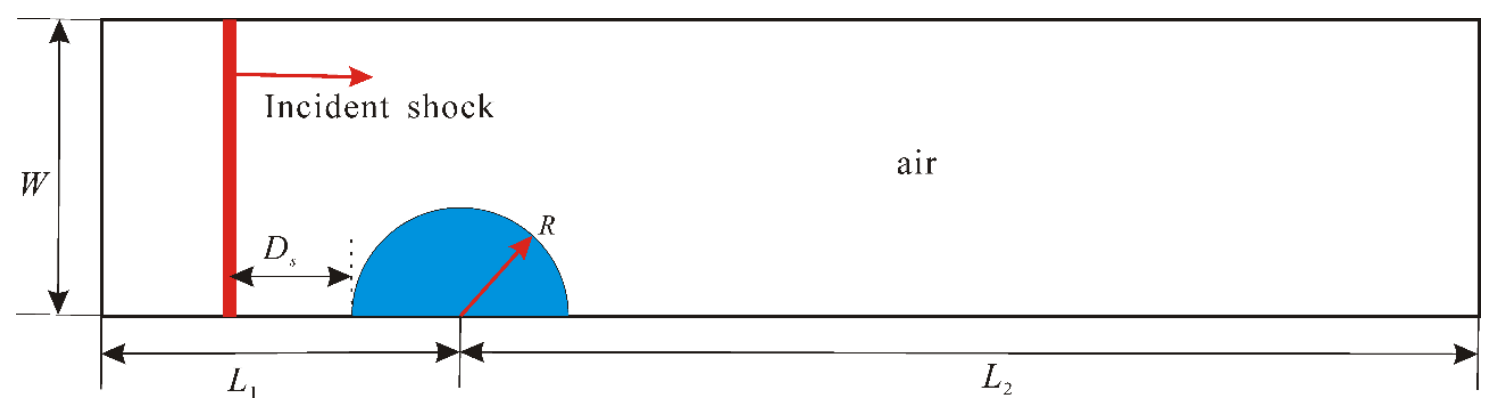

Figure 12. The schematic of the initial setup for the shock-bubble interaction and shock-water-column interaction. 


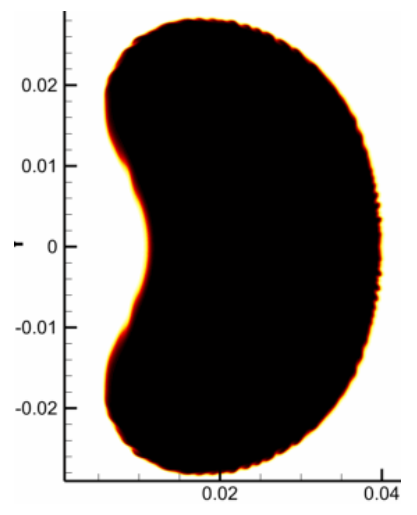

(a) $\mathrm{T}=199.5 \mu \mathrm{s}$

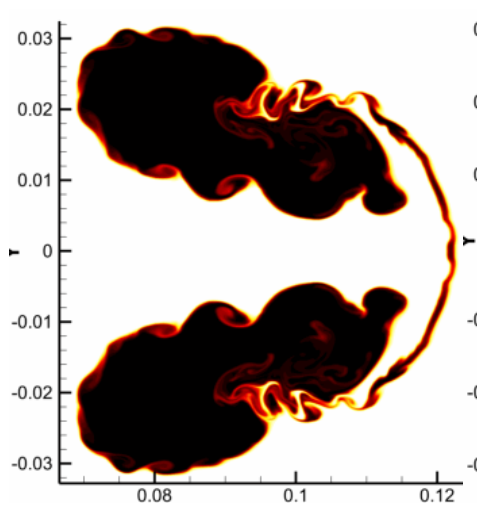

(d) $\mathrm{T}=800.2 \mu \mathrm{s}$

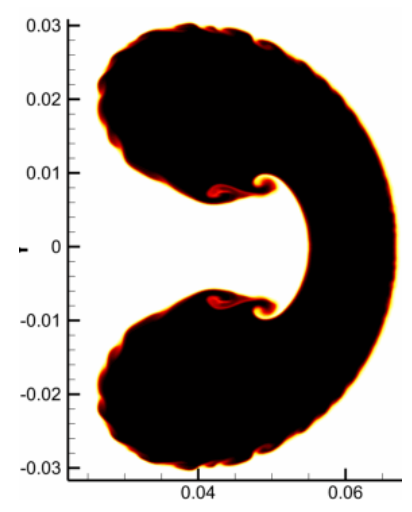

(b) $\mathrm{T}=399.7 \mu \mathrm{s}$

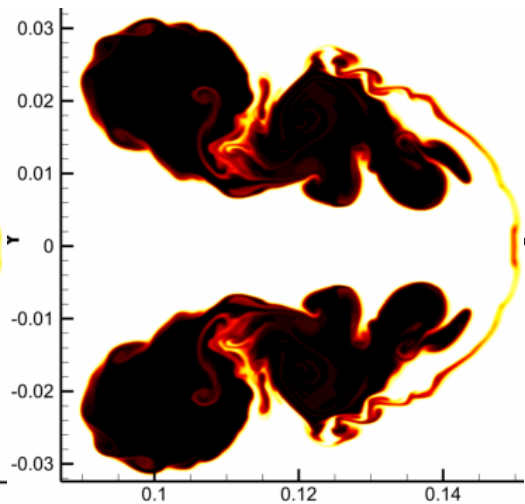

(e) $\mathrm{T}=999.5 \mu \mathrm{s}$

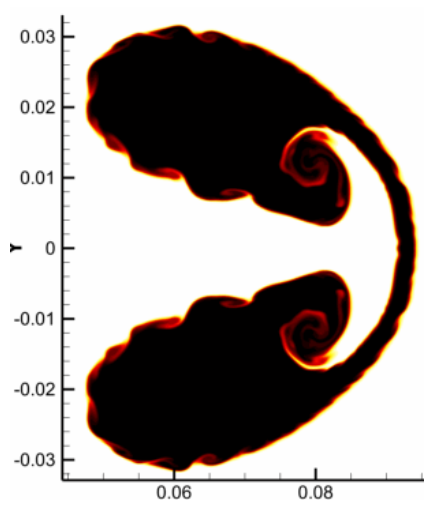

(c) $\mathrm{T}=600.6 \mu \mathrm{s}$

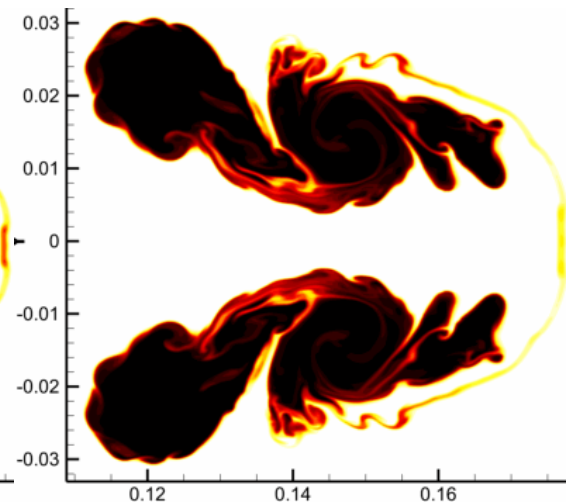

(f) $\mathrm{T}=1200 \mu \mathrm{s}$

Figure 13. Deformation history of the helium bubble impacted by a Mach 1.22 shock.

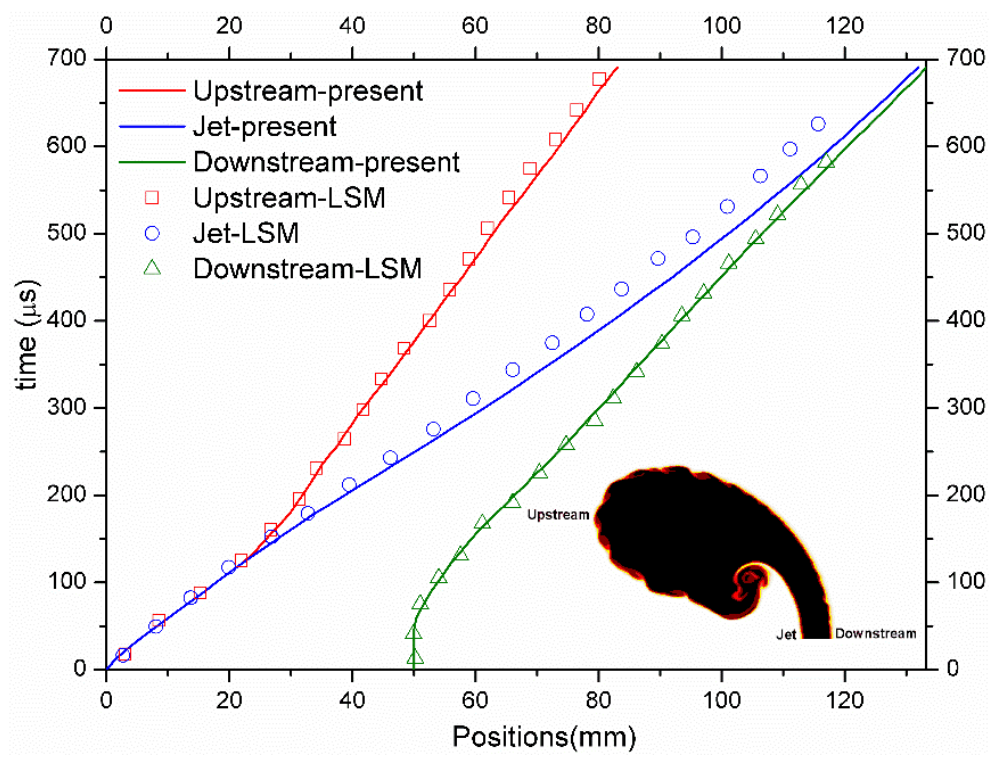

Figure 14. Position history of the helium bubble at the initial stage. 


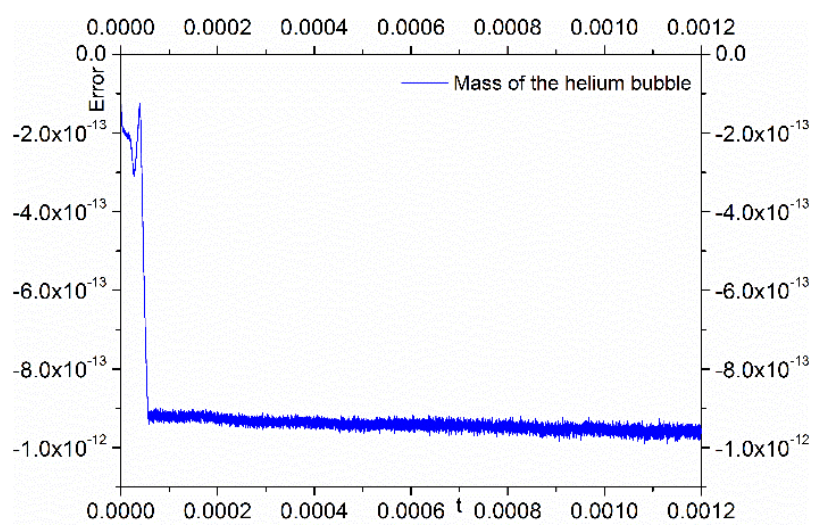

(a)

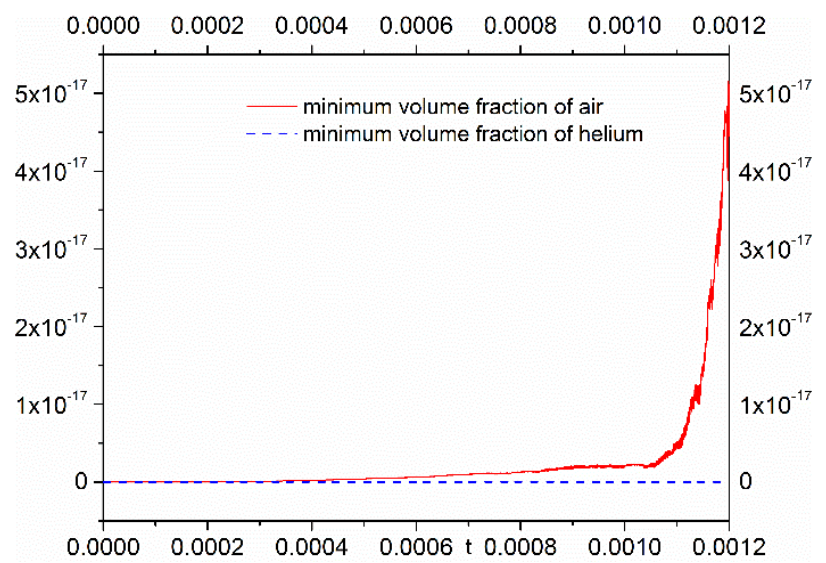

(b)

Figure 15. (a) The errors of the mass of the helium bubble and (b) the histories of minimum volume fractions for the shock-bubble interaction problem.

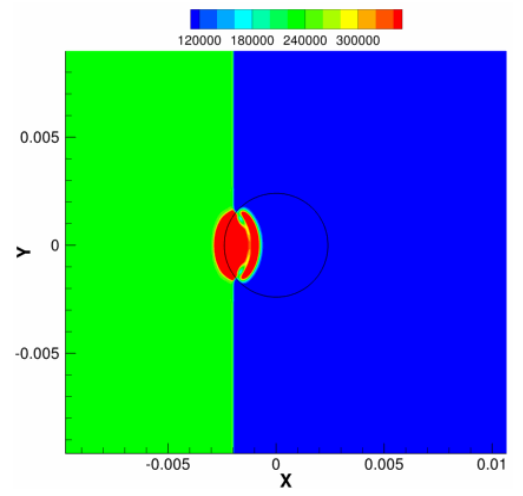

(a) $\mathrm{T}=1.85 \mu \mathrm{s}$

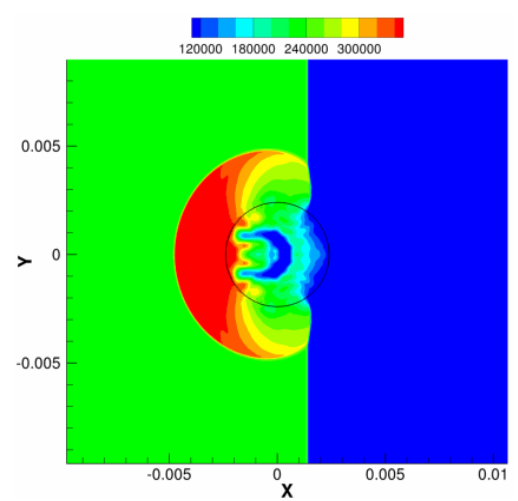

(d) $\mathrm{T}=8.61 \mu \mathrm{s}$

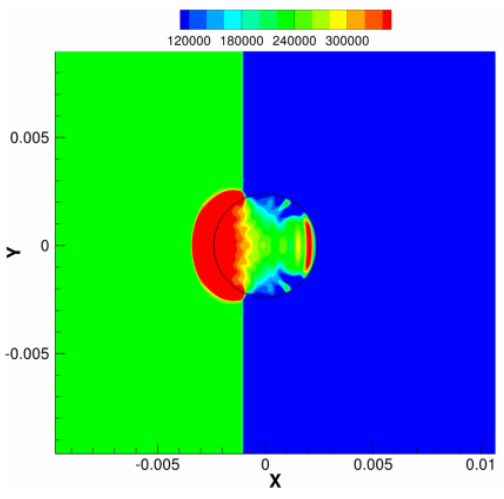

(b) $\mathrm{T}=3.69 \mu \mathrm{s}$

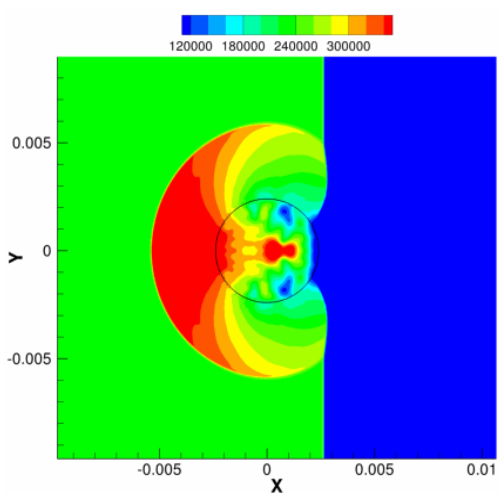

(e) $\mathrm{T}=11.1 \mu \mathrm{s}$

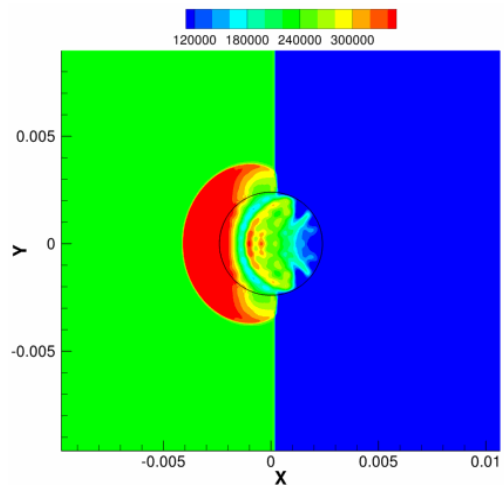

(c) $\mathrm{T}=8.61 \mu \mathrm{s}$

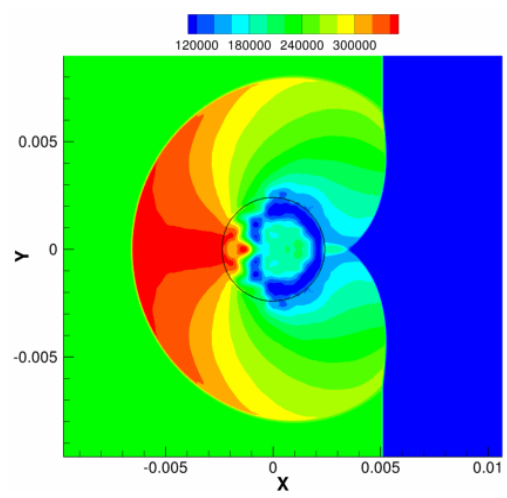

(f) $\mathrm{T}=16 \mu \mathrm{s}$

Figure 16. Sequences of pressure contours at the early stage of shock-water-column interaction (Ms=1.47). 


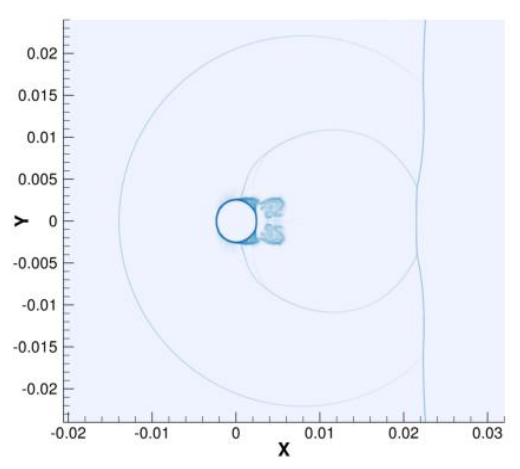

(a) $\mathrm{T}=50.4 \mu \mathrm{s}$

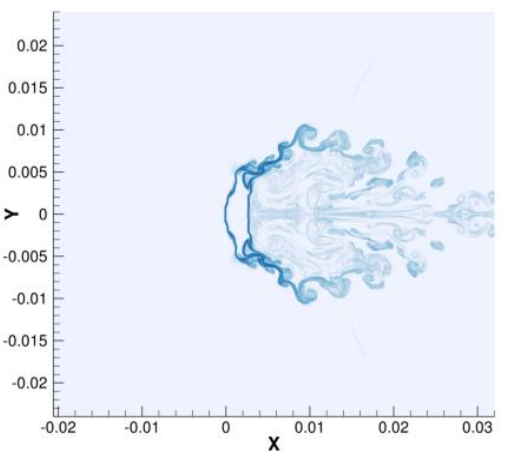

(d) $\mathrm{T}=300.6 \mu \mathrm{s}$

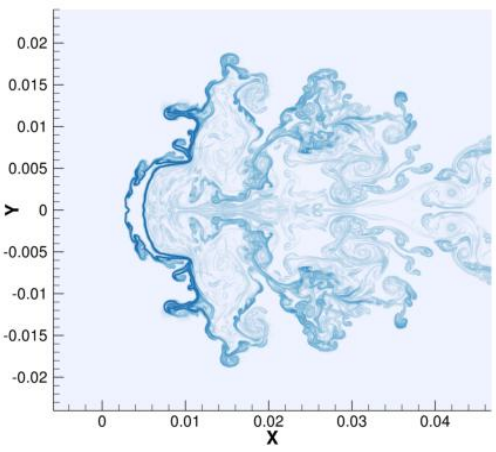

(g) $\mathrm{T}=500.2 \mu \mathrm{s}$

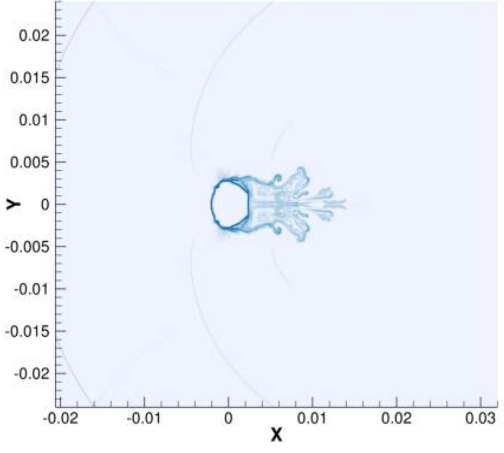

(b) $\mathrm{T}=100.1 \mu \mathrm{s}$

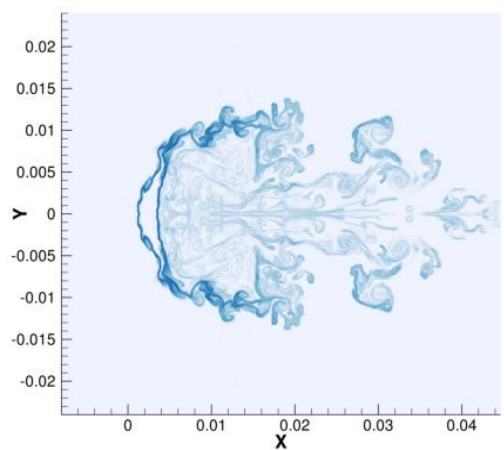

(e) $\mathrm{T}=400.4 \mu \mathrm{s}$

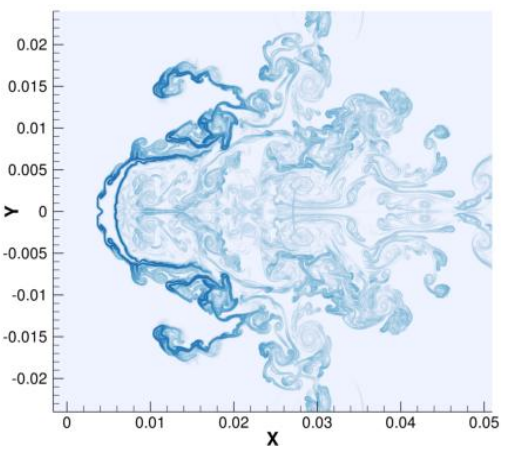

(h) $\mathrm{T}=550.1 \mu \mathrm{s}$

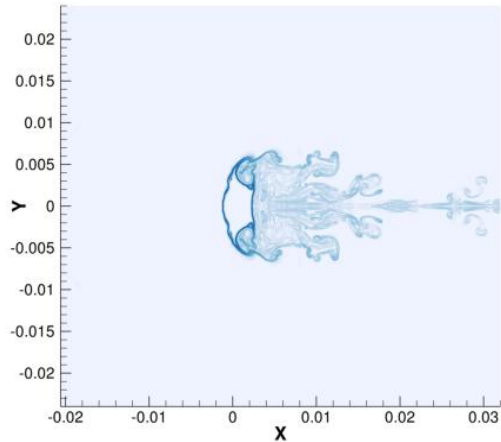

(c) $\mathrm{T}=200.3 \mu \mathrm{s}$

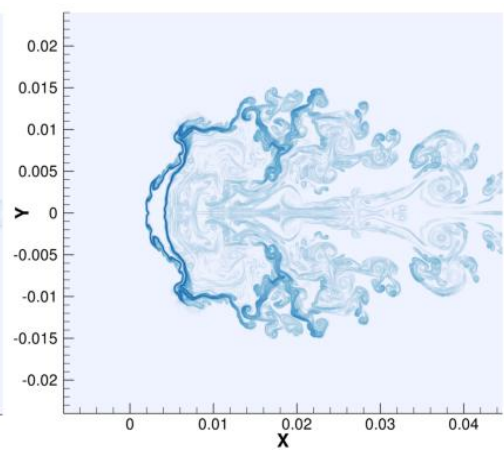

(f) $\mathrm{T}=450 \mu \mathrm{s}$

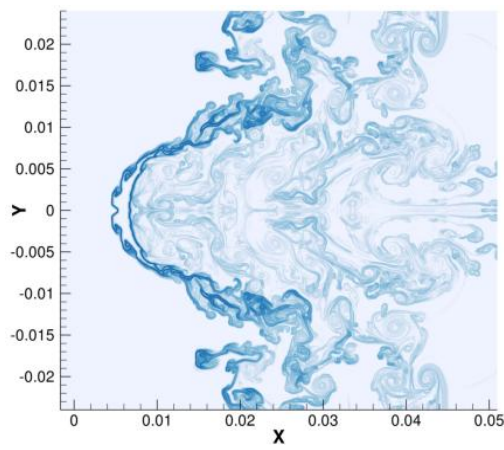

(i) $\mathrm{T}=600.5 \mu \mathrm{s}$

Figure 17. Evolution of the optimized Schlieren plot during the stripping breakup of the water column impacted by a shock $(\mathrm{Ms}=1.47)$. 


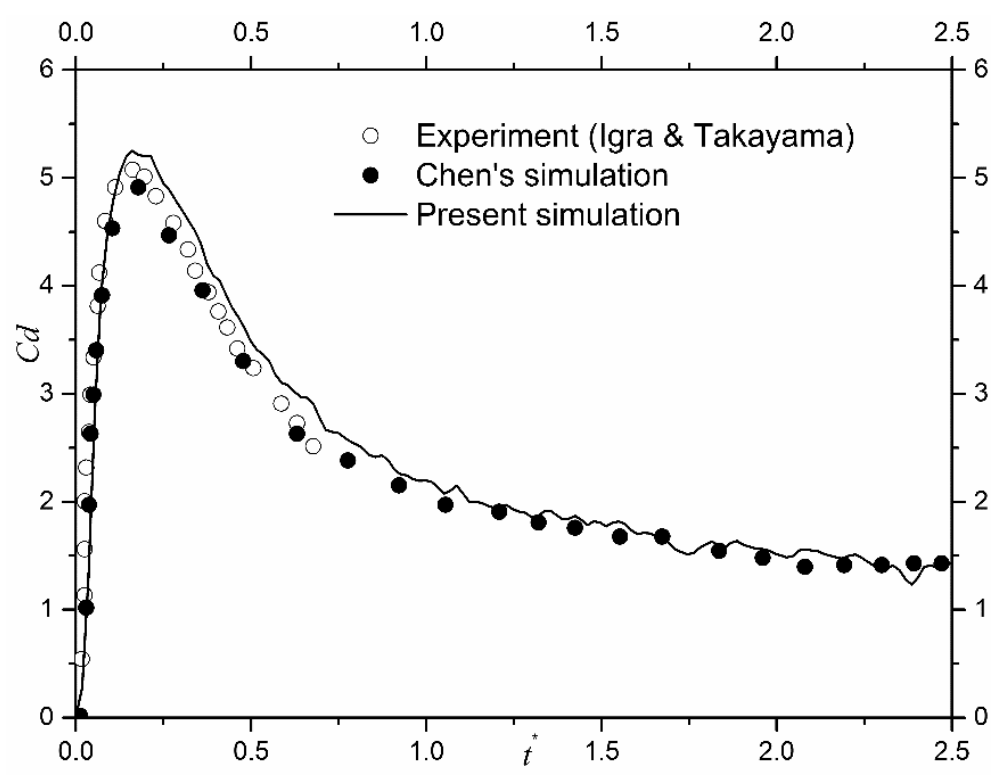

Figure 18. Comparison of the drag coefficient $(C d)$ of a water column impacted by a shock $(\mathrm{Ms}=1.47)$. 


\begin{tabular}{|c|c|c|c|}
\hline Mesh Number & $\mathrm{T}_{1}$ & $\mathrm{~T}_{2}$ & $\left(\mathrm{~T}_{2}-\mathrm{T}_{1}\right) / \mathrm{T}_{1}$ \\
\hline $400 \times 400$ & 898 & $912 \mathrm{~s}$ & $1.6 \%$ \\
\hline $600 \times 600$ & $2999 \mathrm{~s}$ & $3060 \mathrm{~s}$ & $2 \%$ \\
\hline $800 \times 800$ & $7031 \mathrm{~s}$ & $7177 \mathrm{~s}$ & $2.1 \%$ \\
\hline
\end{tabular}

Table 1. Computational costs using different meshes for Example 4. $\mathrm{T}_{1}$ : computational cost without limiter. $\mathrm{T}_{2}$ : computational cost with limiter. 